\title{
Optimal Control Analysis of a Mathematical Model for Breast Cancer
}

\author{
CorrespondingAuthor,E-mail :segunoke2016@gmail.com S.I. Oke
}

M.B. Matadi and S.S. Xulu

Department of Mathematical Sciences,

University of Zululand,

Private Bag X1001, KwaDlangezwa, 3886, South Africa.

Keywords: Breast Cancer, Optimal control, Ketogenic diet, Chemotherapy.

\section{Abstract}

In this paper, a mathematical model of breast cancer governed by a system of ordinary differential equations in the presence of chemotherapy treatment and ketogenic diet is discussed. Several comprehensive mathematical analysis was carried out using varieties of analytical methods to study the stability of the breast cancer model. Also, sufficient conditions on parameter values to ensure cancer persistence in the absence of anti-cancer drugs ketogenic diet and cancer emission when anti-cancer drugs, immune-booster, ketogenic diet are included were established. Furthermore, optimal control theory is applied to find out the optimal drug adjustment as an input control of the system therapies to minimize the number of cancerous cells by considering different controlled combinations of administering the chemotherapy agent and ketogenic diet using the popular Pontryagin's Maximum Principle. Numerical simulations were presented to validate our theoretical results.

\section{Introduction}

Cancer is a generic name that refers to a group of diseases in which normal cells divide uncontrollably, that is grow more rapidly than normal cells, and may eventually spread to other parts of the body by a process called metastasis [6]. According to National Cancer Registry [13], cancer kills more people than TB, AIDs and Malaria combined. Statistics show that cancer related deaths amounted to about 8.2 million in 2010. Mortality rate from cancer is projected to continue to rise with an estimated 13 million death by 2030 [20]. Most common types of cancer include: Breast cancer, Prostrate cancer, Brain cancer, Lung cancer, Skin cancer and so on.

According to the [20] report, breast cancer is the most common invasive cancer in females worldwide. The formation of breast cancer can occur in the inner lining of the milk ducts known as ductal carcinoma or in the lobules of the breast, known as lobular carcinoma [1]. Breast cancer is one of the most widely recognized obstructive diseases in females around the world. The disease has presently been named as the most dangerous cancer in women [20].

However, little is known on the causes of the ailment. There are three major breast cancer risk factors such as hormonal imbalance(estrogen), genetic (family history) and environmental (poor diet,alcohol consumption, smoking, exposure to toxin,etc)[15]. Surgery, Chemotherapy, Radition therapy, Hormonal therapy, Hyperthemia, Targeted therapy and Ketogenic diet $[2,15]$ amongst others therapeutic are used to inhibit the tumor growth or kill the tumor cells in the body. However, each treatment has side effects attributed to it, for example hair loss, vomiting, nausea, fatigue etc. Adverse effects occur as a result of chemotherapy not able to differentiate between normal cells and tumor cells rather sometimes kill both of them [20]. 
Several dietary components and supplements have been examined as possible cancer prevention agents; until recently when few studies such as: $[2,7,18]$ investigated diet as a possible adjuvant to cancer treatment such as ketogenic diet. Ketogenic diet consists of high edible fat with moderate or low protein content and very low carbohydrates, which forces the body to burn fat instead of glucose for adenosine triphosphate (ATP) synthesis [2].

It is well-known that mathematical model is a capable device to investigate the spread of non-infectious diseases; and to provide important insights into diseases behaviour and control $[10,14]$. Over the years it has also become an important tool in comprehending the dynamics of diseases; and in decision making processes regarding medical intervention programs for controlling breast cancer in many nations [4]. For instance,[23] explored the role of mathematical modeling on optimal delivery of combination therapy for tumors and to improve on anti-tumor drugs delivery.

Although, old and recent studies such as $[25,26,1,4]$ amongst others have shown that mathematical modeling is a widely used tools for resolving questions on public health. During the time of Bernoulli's ( on modeling the dynamics of Smallpox) in 1760 [24], Kermack and McKendrick $[25,26]$ and some other recent studies by $[1,4,5,12,22,23]$. These studies reveal that much has not been done in terms of mathematical modeling of nutritional diet (Ketogenic-diet) as a control or therapy on tumor cells. We improved the model in [12] for this paper by incorporating of time dependent control parameters (use of ketogenic diet, immune booster and anti-cancer drugs) based on the assumption that there is interaction between normal cells and tumor cells which is due to mutation in DNA as a result of excess estrogen in the body system $[1,12,19]$.

Furthermore, we analysed and applied optimal control to the improved model to determine the possible impacts of ketogenic-diet use and anti-cancer drugs as a treatment on tumor cells. We carry out rigorous qualitative optimal control analysis of the resulting model and find the necessary conditions for optimal control of the disease using Pontryagin's maximum principle [14] in other to determine optimal strategies for controlling the metastatic of the tumor cells.

This paper is organised as follows: In section 2, four compartments model of ODEs to study the dynamics of breast cancer are developed. In section 3 , the existence of equilibria, their stabilities and basic reproductive number are discussed. In section 4, uncertainty and sensitivity analysis to check the most sensitive parameters in the model were discussed. In section 5 , an optimal control problem according to the model is proposed, an optimal solution is solved. Numerical simulations are illustrated by implementing the forward and backward finite difference scheme in section 6, while concluding remarks are provided in Section 7.

\section{Model formulation}

Based on existing model in [12], we developed our model by assuming logistic (Verhulst) growth of cell population and basis competition between normal cells and tumor cells. We considered the immune cells compartment to comprise of Natural Killer cells (NK) and $C D 8^{+} \mathrm{T}^{-}$ cells as in [12] and we used similar equation to model the immune response dynamic by introducing immune booster (ketone bodies) and anti-cancer drug efficacy. 
We adapted estrogen equation as presented in a model by [16]. Pinho and his coworkers in [16] considered a chemotherapy agent as continuously infused into the body engulfed by different cell populations and natural death can occur. We handled excess estrogen in a similar way and assumed that it is saturated daily through birth control (constant source rate) $(1-k)$ was introduced to served as anti-cancer drug efficacy (e.g Tamoxifen) in order to bind estrogen receptors positive and to reduce excess estrogen from promoting cancer growth [3].

In this study, we reflected on the model that splits the entire population of cells of the human breast tissues at any given periods of time $P(t)$. We shall posit further by describing each compartment, starting with normal cells compartment, represented by $N(t)$ in the form of epithelial cells that constitute the breast tissue. The cells developed and die normally as they have unaltered DNA which controls all cell activities. We assumed that the normal cells and tumor cells compete for nutrients and other resources in a small volume and hence assumed a competition model used by Gateby, (1995).

$$
\frac{d N}{d t}=N \alpha_{1}-\mu_{1} N^{2}-\phi_{1} N T-(1-k) \lambda_{1} N E
$$

The first term represents the logistic growth rate $\alpha_{1}$ of the normal cells, which are breast tissues that are made-up of epithelial cells. The second term represents natural death rate of normal cells, $\phi_{1}$ represent the rate at which normal cells is inhibition due to alteration in DNA that responsible for cancer cells to have uncontrolled cycle than normal cells [12]. The final term describes gene transactivation that can be a contributing growth factor responsible for estrogen stimulated of breast cancer, which can result into damage of DNA. Thus, there will be reduction in the population of normal cells $N(t)=$ being transformed into tumor cells by $\lambda_{1} N E$ where $\lambda_{1}$ represents tumor formation rate resulting from DNA mutation caused by the presence of excess estrogen [1]. However, $(1-k)$ represents effectiveness of anti-cancer drugs (Tamoxifen).

The tumor cells compartment can be denoted by $T(t)$ in the form an abnormal mass of tissue. Tumors are a classic sign of inflammation, and can be benign or malignant ( cancerous). Their names usually reflect the kind of tissue they arise. For example breast cancer, with altered DNA. There are about 51 breast cancer cell lines which mirror the 145 primary breast tumors (Neves and Brindle, 2006). These can be classified into two malor branches, the luminal, which has estrogen receptors $(E S R 1+v e)$ and basal-like without estrogen receptors $(E S R 1-v e)$. We then assume a homogeneous luminal type of cancer cells in form of MDAMB361, MCF-7, BT474, T47D and ZR75 cell lines [12].

$$
\frac{d T}{d t}=T \alpha_{2} d-\mu_{2} T^{2}-\gamma_{2} M T-\mu_{5} T+(1-k) \lambda_{1} N E
$$

The first term of the equation is a limited growth term for tumor cells which depends on the rate of parameter $d$ (ketogenic diets). Although, if $d=0$, automatically tumor cells are eradicated, but any DNA mutation that is caused by excess estrogen will repopulate tumor cells again $\lambda_{1} N E$. The induced death rate $\mu_{5}$ is as a result of tumor starvation of nutrients, glucose and so on from body system during nutrition altered by ketogenic diet. We assume that $\gamma_{2}$ is the rate at which tumor cells are being removed due to effectiveness of immune response. 
The immune response compartment is represented by $M(t)$ inform of Natural Killer (NK) cells and $\mathrm{CD8}^{+}$T cells. Their growth may be stimulated by the presence of the tumor and they can destroy tumor cells through a kinetics process. We also assume that thepresence of a detectable tumor in a body system does not necessarily imply that the tumor has completely escaped active immunosurveillance. However, a tumor is immunogenic, it is possible that the immune response may not be sufficient on its own to completely combat the rapid growth of the tumor cells population and the eventual development into a tumor.

$$
\frac{d M}{d t}=s \beta+\frac{\rho M T}{\omega+T}-\gamma_{3} M T-\mu_{3} M-\left((1-k) \frac{\lambda_{3} M E}{g+E}\right)
$$

The constant source parameter $s$ denotes the source rate of immune response fully infused in the body daily and we introduced immune booster $\beta$ (supplement such as ketone bodies) to assist immune response whenever tumor cells overpower immune cells in order to activate the immune response and fight the cancer cells. The next term is a nonlinear growth term for immune response where $\rho$ is the rate of immune response and $\omega$ is the immune cell threshold [4]. We denoted $\gamma_{3}$ as the rate at which immune response is inactivated upon interacting with tumor cells while $\mu_{3}$ represents immune cells natural death rate as a result of necrosis. The final term explains a limited rate at which estrogen suppresses immune cells activation where $\lambda_{3}$ is the rate of immune suppression and $g$ is the estrogen threshold [12].

Finally, we considered estrogen compartment denoted by $E(t)$, estrogen is a female steroid hormone that is produced by the ovaries in lesser amounts; by the adrenal cortex, plancenta and male testes. Estrogen helps to control and guide sexual development, including the physical changes associated with puberty $[14,17]$. However, increased in estrogen levels can lead to the growth of the tumor cells. It also serve as a mitogen by triggering cell division in breast tissue [17]. Estrogen acts as a carcinogen by directly damaging DNA, making healthy epithelial cells to have a higher likelihood of malignant conversion $[15,17]$.

$$
\frac{d E}{d t}=(1-k) \varepsilon-\mu_{4} E
$$

The constant replenished of excess estrogen is denoted by $\varepsilon$. We assumed that majority of cancer cells are estrogen-receptor positive and only small proportion of epithelial cells are estrogen-receptor positive which can only be blocked by anti-cancer drug (1-k) Tamoxifen. $\mu_{4}$ is the rate at which estrogen is being washed out from the body system. Thus, system (5) is our modified model.

$$
\begin{aligned}
& \frac{d N}{d t}=N\left(\alpha_{1}-\mu_{1} N-\phi_{1} T\right)-(1-k)\left(\lambda_{1} N E\right) \\
& \frac{d T}{d t}=T\left(\alpha_{2} d-\mu_{2} T\right)-\gamma_{2} M T-\mu_{5} T+(1-k)\left(\lambda_{1} N E\right) \\
& \frac{d M}{d t}=s \beta+\frac{\rho M T}{\omega+T}-\gamma_{3} M T-\mu_{3} M-\left((1-k) \frac{\lambda_{3} M E}{g+E}\right) \\
& \frac{d E}{d t}=(1-k) \varepsilon-\mu_{4} E
\end{aligned}
$$


Table 1: Description of parameters in the model

\begin{tabular}{|c|c|c|c|c|}
\hline Parameter & Symbol & Value & Units & Refs \\
\hline \begin{tabular}{|c|} 
Per capita \\
growth rate of \\
normal cells \\
\end{tabular} & $\alpha_{1}$ & 0.70 & $d a y^{-1}$ & [12] \\
\hline \begin{tabular}{|c|} 
Per capita \\
growth rate of \\
tumor cells \\
\end{tabular} & $\alpha_{2}$ & 0.514 & $d a y^{-1}$ & {$[5]$} \\
\hline $\begin{array}{c}\text { Natural death } \\
\text { rate of normal } \\
\text { cells }\end{array}$ & $\mu_{1}$ & 0.00003 & $d a y^{-1}$ & Assumed \\
\hline $\begin{array}{c}\text { Natural death } \\
\text { rate of tumor } \\
\text { cells }\end{array}$ & $\mu_{2}$ & 0.01 & $d a y^{-1}$ & [7] \\
\hline \begin{tabular}{|c|} 
Rate of \\
inhibition of \\
normal cells \\
\end{tabular} & $\phi_{1}$ & $6 \times 10^{-8}$ & $d a y^{-1}$ & [1] \\
\hline $\begin{array}{c}\text { Tumor cells } \\
\text { death rate due } \\
\text { to immune } \\
\text { response }\end{array}$ & $\gamma_{2}$ & $3 \times 10^{-6}$ & $d a y^{-1}$ & [12] \\
\hline $\begin{array}{c}\text { Interaction } \\
\text { coefficient rate } \\
\text { with immune } \\
\text { response }\end{array}$ & $\gamma_{3}$ & $1 \times 10^{-7}$ & $d a y^{-1}$ & [5] \\
\hline $\begin{array}{l}\text { Source rate of } \\
\text { immune cells }\end{array}$ & $s$ & $1.3 \times 10^{4}$ & $d a y^{-1}$ & [12] \\
\hline $\begin{array}{c}\text { Source rate of } \\
\text { estrogen }\end{array}$ & $\varepsilon$ & $1.3 \times 10^{4}$ & $d a y^{-1}$ & est \\
\hline \begin{tabular}{|c|} 
Immune \\
threshold rate
\end{tabular} & $\omega$ & $3 \times 10^{5}$ & $d a y^{-1}$ & [5] \\
\hline $\begin{array}{c}\text { Immune } \\
\text { response rate }\end{array}$ & $\rho$ & 0.20 & $d a y^{-1}$ & [13] \\
\hline $\begin{array}{l}\text { Natural death } \\
\text { rate of } \\
\text { immune cells }\end{array}$ & $\mu_{3}$ & 0.29 & $d a y^{-1}$ & [5] \\
\hline $\begin{array}{c}\text { Efficacy of } \\
\text { anti-cancer } \\
\text { drug }\end{array}$ & $k$ & $0-1$ & $d a y^{-1}$ & Assumed \\
\hline $\begin{array}{c}\text { Supplement } \\
\text { for immune } \\
\text { booster }\end{array}$ & $\beta$ & 0.01 & $d a y^{-1}$ & est \\
\hline \multicolumn{2}{|c|}{ Tumor formation rate as a } & & & \\
\hline
\end{tabular}




\begin{tabular}{|c|c|c|c|c|}
\hline \multicolumn{2}{|c|}{ result } & \multicolumn{2}{c|}{ est } \\
\hline $\begin{array}{c}\text { of DNA } \\
\text { damage by } \\
\text { excess } \\
\text { estrogen }\end{array}$ & $\lambda_{1}$ & 0.20 & $(P g / m L)^{-1} d a y^{-}$ & est \\
\hline $\begin{array}{c}\text { Immune } \\
\text { suppression } \\
\text { rate due to } \\
\text { excess } \\
\text { estrogen }\end{array}$ & $\lambda_{3}$ & 0.002 & $d a y^{-1}$ & \\
\hline $\begin{array}{c}\text { Assume } \\
\text { constant of } \\
\text { value of decay } \\
\text { factor }\end{array}$ & $g$ & 0.1 & $d a y^{-1}$ & est \\
\hline $\begin{array}{c}\text { Natural death } \\
\text { rate of } \\
\text { estrogen }\end{array}$ & $\mu_{4}$ & 0.97 & $d a y^{-1}$ & [19] \\
\hline $\begin{array}{c}\text { Death rate due } \\
\text { to ketogenic } \\
\text { diet }\end{array}$ & $\mu_{5}$ & 2.0 & $d a y^{-1}$ & est \\
\hline $\begin{array}{c}\text { Constant rate } \\
\text { of ketogenic } \\
\text { diet }\end{array}$ & $d$ & 0.5 & day $^{-1}$ & est \\
\hline
\end{tabular}

\section{Model Analysis}

\subsection{Boundedness and Positivity of solutions}

The system of equations (5) has an initial condition by

$N(0)=N_{0} \geq 0, \quad T(0)=T_{0} \geq 0, \quad M(0)=M_{0} \geq 0, \quad$ and $\quad E(0)=E_{0} \geq 0$

since our model is to investigate cellular populations, therefore all the variables and parameters of the model are non-negative. Based on biological finding, the system of equations(5) will be studies in the following region such as :

$$
\Delta=\left\{(N, T, M, E) \in \mathfrak{R}_{+}^{4}\right\}
$$

The following theorem assures that the system of equations(5) is well-posed such that solutions with non-negative initial conditions remain non-negative for all $0<t<\infty$, and therefore makes the variable biologically meaningful. Hence, we have the following result: 
Theorem 1. : The region $\Delta \subset \mathfrak{R}_{+}^{4}$ is positively invariant with respect to the system of equations(5) and non-negative solution exists for all time $0<t<\infty$.

Proof: Let $\Delta=\Delta_{c} \subset \mathfrak{R}_{+}^{4}$ with $\Delta=\left\{(N, T, M, E) \in \mathfrak{R}_{+}^{4}: N \leq \frac{\alpha_{1}}{\mu_{1}}\right\}$

then the solutions ( $\mathrm{N}(\mathrm{t}), \mathrm{T}(\mathrm{t}), \mathrm{M}(\mathrm{t}), \mathrm{E}(\mathrm{t}))$ of system (5) are positive $\forall t \geq 0$. It is obvious from the first compartment of system (5) that

$$
\frac{d N}{d t} \leq N(t) \alpha_{1}-\mu_{1} N^{2}(t)
$$

solving with Bernoulli method and take $N(0)=N_{0}$,

we have,

$$
N(t) \leq \frac{\alpha_{1}}{\mu_{1}+k \alpha_{1} e^{-\alpha_{1} t}}
$$

with

$$
\begin{gathered}
k=\frac{\alpha_{1}-N_{0} \mu_{1}}{N_{0} \alpha_{1}} \\
N_{0}=\frac{\alpha_{1}}{\mu_{1}+k \alpha_{1}}
\end{gathered}
$$

Then,

$$
\begin{gathered}
N(t) \leq \frac{\alpha_{1}}{\mu_{1}+\left(\frac{\alpha_{1}-N_{0} \mu_{1}}{N_{0}}\right) e^{-} \alpha_{1} t} \\
N(t) \leq \frac{\alpha_{1}}{\mu_{1}} \text { as } t \rightarrow \infty
\end{gathered}
$$

hence, $N(t)>0, \forall t>0$.

Consequently, it can be shown that $T(t)>0, M(t)>0$, and $E(t)>0 \forall t>0$. This complete the proof.

\subsection{The equilibrium points of system (5)}

The steady states occur by setting the LHS of system (5) to equal to zero, i.e.

$$
\frac{d N}{d t}=\frac{d T}{d t}=\frac{d M}{d t}=\frac{d E}{d t}=0
$$

The model system admits six steady states in which there are four dead equilibria, one tumor free equilibrium points and one co-existing equilibrium point $P=\left(N^{*}, T^{*}, M^{*}, E^{*}\right)$

where; $N^{*}, T^{*}, M^{*}, E^{*}$ represent the tumor-free equilibrium values for the normal cells, 
tumor cells, immune cells and estrogen hormone respectively. We have $N^{*}>0, M^{*}>0$, $E^{*}>0$ since cell populations are non-negative and real. Therefore, all parameters $\mathrm{s}$, $\beta, g, \mu_{1}, \mu_{3}, \mu_{4}, \varepsilon, \lambda_{3}, k, \alpha_{1}$, and $\lambda_{1}$ are positive.

Tumor-Free Equilibrium point

$$
P_{0}=\left(\frac{\alpha_{1} \mu_{4}-(1-k)^{2} \lambda_{1} \varepsilon}{\mu_{1} \mu_{4}}, 0, \frac{s \beta\left(g \mu_{4}+(1-k) \varepsilon\right)}{\mu_{3}\left(g \mu_{4}+(1-k) \varepsilon\right)+(1-k)^{2} \lambda_{3} \varepsilon}, \frac{(1-k) \varepsilon}{\mu_{4}}\right)
$$

Type 1 Dead equilibrium point

$$
P_{d 1}=\left(0,0, \frac{s \beta\left(g \mu_{4}+(1-k) \varepsilon\right)}{\left.\mu_{3}\left(g \mu_{4}+(1-k) \varepsilon\right)+(1-k)^{2} \lambda_{3} \varepsilon\right)}, \frac{(1-k) \varepsilon}{\mu_{4}}\right)
$$

Type 2 Dead equilibrium point

$$
P_{d 2}=\left(0, \frac{d \alpha_{2}-\gamma_{2} m_{1}^{*}-\mu_{5}}{\mu_{2}}, m_{1}^{*}, \frac{(1-k) \varepsilon}{\mu_{4}}\right)
$$

Type 3 Dead equilibrium point

$$
P_{d 3}=\left(0, \frac{d \alpha_{2}-\gamma_{2} m_{2}^{*}-\mu_{5}}{\mu_{2}}, m_{2}^{*}, \frac{(1-k) \varepsilon}{\mu_{4}}\right)
$$

Type 4 Dead equilibrium point

$$
P_{d 4}=\left(0, \frac{d \alpha_{2}-\gamma_{2} m_{3}^{*}-\mu_{5}}{\mu_{2}}, m_{3}^{*}, \frac{(1-k) \varepsilon}{\mu_{4}}\right)
$$

co-existing equilibrium points

$$
P_{e}=\left(N_{4}^{*}, T_{4}^{*}, M_{4}^{*}, E_{4}^{*}\right)
$$

\subsection{The Reproductive number and Tumor-free equilibrium point}

In this section, we mainly analysed the stability behaviours of system (5) by means of eigenvalues. We apply Hartman Grobman Theorem which states that in the neighbourhood of a hyperbolic equilibrium point, a nonlinear dynamical system is topologically equivalent to its linearisation [21]

Theorem 2. : The Tumor-free equilibrium point $P_{0}$ of system (5) is locally asymptotically stable if $R_{0}<1$, otherwise unstable.

Proof: Linearising system (5) around TFE $P_{0}$, we obtained the following Jacobian matrix $J\left(P_{0}\right)$.

$$
J=\left(\begin{array}{cccc}
\frac{2 \mu_{1} \lambda_{1}(1-k)^{2} \varepsilon-\alpha_{1} \mu_{1} \mu_{4}-(1-k)^{2} \lambda_{1} \mu_{1} \varepsilon}{\mu_{1} \mu_{4}} & \frac{(1-k)^{2} \lambda_{1} \Phi_{1} \varepsilon-\Phi_{1} \alpha_{1} \mu_{4}}{\mu_{1} \mu_{4}} & 0 & -B_{6} \\
\frac{(1-k)^{2} \lambda_{1} \varepsilon}{\mu_{4}} & B_{3} & 0 & B_{6} \\
0 & B_{4} & -B_{5} & -B_{7} \\
0 & 0 & 0 & -\mu_{4}
\end{array}\right)
$$




$$
\begin{gathered}
J\left(P_{0}\right)=\left(\begin{array}{cccc}
B_{0} & B_{2} & 0 & -B_{6} \\
B_{1} & B_{3} & 0 & B_{6} \\
0 & B_{4} & -B_{5} & -B_{7} \\
0 & 0 & 0 & -\mu_{4}
\end{array}\right) \\
\left|J\left(P_{0}\right)\right|=\left|\begin{array}{cccc}
B_{0}-\delta & B_{2} & 0 & -B_{6} \\
B_{1} & B_{3}-\delta & 0 & B_{6} \\
0 & B_{4} & -B_{5}-\delta & -B_{7} \\
0 & 0 & 0 & -\mu_{4}-\delta
\end{array}\right|=0
\end{gathered}
$$

Then the characteristic equation at $P_{0}$ of the linearised system of the model (5) is given below. Obviously, there exists two negative characteristic roots

$$
\delta_{1}=-\mu_{4}, \quad \delta_{2}=-B_{5}
$$

However, we only need to consider

$$
\begin{gathered}
\delta^{2}-\left(B_{0}+B_{3}\right) \delta+B_{0} B_{3}-B_{1} B_{2}=0 \\
\delta^{2}-\left(B_{0}+B_{3}\right) \delta+B_{0} B_{3}\left(1-\frac{B_{1} B_{2}}{B_{0} B_{3}}\right)=0
\end{gathered}
$$

where our basic reproduction number is

$$
\begin{gathered}
R_{0}=\frac{B_{1} B_{2}}{B_{0} B_{3}} \\
\delta^{2}-\left(B_{0}+B_{3}\right) \delta+B_{0} B_{3}\left(1-R_{0}\right)=0,
\end{gathered}
$$

where;

$$
B_{0}=\frac{2 \mu_{1} \lambda_{1}(1-k)^{2} \varepsilon-\alpha_{1} \mu_{1} \mu_{4}-(1-k)^{2} \lambda_{1} \mu_{1} \varepsilon}{\mu_{1} \mu_{4}}, B_{1}=\frac{(1-k)^{2} \lambda_{1} \varepsilon}{\mu_{4}}, B_{2}=\frac{(1-k)^{2} \lambda_{1} \Phi_{1} \varepsilon-\Phi_{1} \alpha 1 \mu_{4}}{\mu_{1} \mu 4}
$$

$$
B_{3}=\frac{\alpha_{2} \mu_{3} d\left(g \mu_{4}+(1-k) \varepsilon\right)+\alpha_{2} \lambda_{3} d \varepsilon(1-k)^{2}-\gamma_{2} s \beta\left(g \mu_{4}+(1-k) \varepsilon\right)-\mu_{5} \mu_{3}\left(g \mu_{4}+(1-k) \varepsilon\right)-\mu_{5} \lambda_{3} \varepsilon(1-k)^{2}}{\mu_{3}\left(g \mu_{4}+(1-k) \varepsilon\right)+(1-k)^{2} \lambda_{3} \varepsilon}
$$


$B_{6}=\frac{(1-k) \lambda_{1} \alpha_{1} \mu_{4}-(1-k)^{3} \lambda_{1}^{2} \varepsilon}{\mu_{1} \mu_{4}},-B_{7}=-\left(\frac{\mu_{4}^{2}(1-k) \lambda_{3} g s \beta\left(g \mu_{4}+(1-k) \varepsilon\right)}{\left(g \mu_{4}+(1-k) \varepsilon\right)^{2}\left[\mu_{3}\left(g \mu_{4}+(1-k) \varepsilon\right)+(1-k)^{2} \lambda_{3} \varepsilon\right]}\right)$

Here, we can apply the Routh-Hurwitz criterion namely.

(i) $\operatorname{Tr}(A)<0 \quad$ (ii) $\operatorname{Det}(A)>0$

provided;

$a_{0}=1>0, \quad a_{1}=\left(B_{0}+B_{3}\right)<0, \quad B_{0} B_{3}\left(1-R_{0}\right)>0 \quad$ if $R_{0}<1$,

$B_{0} B_{3}>B_{1} B_{2}, \quad B_{1}>0, \quad B_{2}>0, \quad B_{3}<0, \quad B_{0}<0$

Since Routh - Hurwitz criterion hold, all the eigenvalues are negative i.e. $\delta_{3}<0$ and $\delta_{4}<0$.

Therefore, the TFE point of system (5) is locally asymptotically stable if $R_{0}<1$ otherwise unstable.

The epidemiological implication of the above result is that the tumor cells that are governed by system (5) can be eliminated from the population (normal cells or breast tissues) whenever an influx by tumor cells into the normal cells is small such that $R_{0}<1$. Therefore, the existence of a tumor-free equilibrium in this case depends on the estrogen level.

Theorem 3 : The Type 1 Dead equilibrium point $P_{d 1}$ of system (5) is locally asymptotically stable if

$$
\left(\frac{(1-k)^{2} \lambda_{1} \varepsilon}{\alpha_{1} \mu_{4}}\right)>1
$$

, otherwise unstable.

Proof : Linearising system (5) around the Dead free equilibrium point $P_{d 1}$, we obtained the following Jacobian matrix $J\left(P_{d 1}\right)$

$$
J=\left(\begin{array}{cccc}
\alpha_{1}-(1-k) \lambda_{1} E_{0}^{*} & 0 & 0 & 0 \\
(1-k) \lambda_{1} E_{0}^{*} & d \alpha_{2}-\gamma_{2} M_{0}^{*}-\mu_{5} & 0 & 0 \\
0 & \frac{\rho \omega M_{0}^{*}-\gamma_{3} M_{0}^{*} \omega^{2}}{\omega^{2}} & -\left(\frac{\mu_{3}\left(g+E_{0}^{*}\right)+(1-k) \lambda_{3} E_{0}^{*}}{\left(g+E_{0}^{*}\right)}\right) & \frac{\lambda_{3} g M_{0}^{*}(1-k)}{\left(g+E_{0}^{*}\right)^{2}} \\
0 & 0 & 0 & -\mu_{4}
\end{array}\right)
$$




$$
\begin{gathered}
J\left(P_{d 1}\right)=\left(\begin{array}{cccc}
B_{0} & 0 & 0 & 0 \\
B_{1} & B_{2} & 0 & 0 \\
0 & B_{3} & -B_{4} & -B_{5} \\
0 & 0 & 0 & -\mu_{4}
\end{array}\right) \\
\left|J\left(P_{1}\right)\right|=\left|\begin{array}{cccc}
B_{0}-\delta & 0 & 0 & 0 \\
B_{1} & B_{2}-\delta & 0 & 0 \\
0 & B_{3} & -B_{4}-\delta & -B_{5} \\
0 & 0 & 0 & -\mu_{4}-\delta
\end{array}\right|=0
\end{gathered}
$$

Clearly, two eigenvalues of the system (5) at $P_{d 1}$ are negative and real $\delta_{1}=-\mu_{4} \quad$ and

$$
\delta_{2}=-B_{4} \Rightarrow-\left(\frac{\mu_{3}\left(g \mu_{4}+(1-k) \varepsilon\right)+(1-k)^{2} \lambda_{3} \varepsilon}{g \mu_{4}+(1-k) \varepsilon}\right)
$$

While the remaining two eigenvalues are obtained from $2 \times 2$ matrix.

$$
A=\left(\begin{array}{cc}
B_{0} & 0 \\
B_{1} & B_{2}
\end{array}\right)
$$

Applying the Routh-Hurwitz criterion stated above; we have

(i) $\operatorname{Tr}(A)=B_{0}+B_{2}$

$$
\Rightarrow\left(\frac{\alpha_{1} \mu_{4}-(1-k)^{2} \lambda_{1} \varepsilon}{\mu_{4}}+\frac{\left(\mu_{3} \alpha_{2} d-\gamma_{2} s \beta-\mu_{5} \mu_{3}\right) A^{*}+d \alpha_{2} \lambda_{3}(1-k)^{2} \varepsilon-(1-k)^{2} \lambda_{3} \mu_{5} \varepsilon}{\mu_{3} A^{*}+(1-k)^{2} \lambda_{3} \varepsilon}\right)<0
$$

$$
\text { if } \alpha_{1}\left(1-\frac{(1-k)^{2} \lambda_{1} \varepsilon}{\alpha_{1} \mu_{4}}\right)>0, \Rightarrow\left(\frac{(1-k)^{2} \lambda_{1} \varepsilon}{\alpha_{1} \mu_{4}}\right)>1
$$

Therefore, $\operatorname{Tr}(A)<0$

ii) $\operatorname{Det}(A)=B_{0} B_{2}$

$$
\left(\left(\frac{\alpha_{1} \mu_{4}-(1-k)^{2} \lambda_{1} \varepsilon}{\mu_{4}}\right)\left(\frac{\left(\mu_{3} \alpha_{2} d-\gamma_{2} s \beta-\mu_{5} \mu_{3}\right) A^{*}+d \alpha_{2} \lambda_{3}(1-k)^{2} \varepsilon-(1-k)^{2} \lambda_{3} \mu_{5} \varepsilon}{\mu_{3} A^{*}+(1-k)^{2} \lambda_{3} \varepsilon}\right)\right)>0
$$

$$
\text { if } \alpha_{1}\left(1-\frac{(1-k)^{2} \lambda_{1} \varepsilon}{\alpha_{1} \mu_{4}}\right)>0 \text { provided }\left(\frac{(1-k)^{2} \lambda_{1} \varepsilon}{\alpha_{1} \mu_{4}}\right)>1
$$


and

$$
\left\{\left(\alpha_{1}\left(1-\frac{(1-k)^{2} \lambda_{1} \varepsilon}{\alpha_{1} \mu_{4}}\right)\right)\left(\left(\frac{\left(\mu_{3} \alpha_{2} d-\gamma_{2} s \beta-\mu_{5} \mu_{3}\right) A^{*}+d \alpha_{2} \lambda_{3}(1-k)^{2} \varepsilon-(1-k)^{2} \lambda_{3} \mu_{5} \varepsilon}{\mu_{3} A^{*}+(1-k)^{2} \lambda_{3} \varepsilon}\right)\right)\right\}>0
$$

implies that $\operatorname{Det}(A)>0$. Thus, the remaining eigenvalues $\delta_{3}$ and $\delta_{4}$ are negative and real since R-H Criterion has been satisfied. Hence, the type 1-Dead equilibrium point $P_{d 1}$ of the system (5) is locally asymptotically stable if $\left(\frac{(1-k)^{2} \lambda_{1} \varepsilon}{\alpha_{1} \mu_{4}}\right)>1$.

Epidemiologically implies that the net growth of the tumor cells must be more than the immune cells values in order to have the tumor cells overpower the normal cells as the reactivation of the immune cells due to estrogen effects that are greater than the reactivation of the immune cells due to tumor effect. However, ketogenic diet is inactive at type 1-Dead equilibrium point.

Theorem 4 : The Type 2 Dead equilibrium point $P_{d 2}$ of system (5) is locally asymptotically stable if

$$
\begin{gathered}
\left(\frac{(1-k)^{2} \lambda_{1} \varepsilon}{\alpha_{1} \mu_{4}}\right)>1 \\
\omega>\frac{A^{*}}{\mu_{2}}\left(\frac{\mu_{2} C^{*} \rho}{\gamma_{3} A^{*} C^{*}+\mu_{2} \mu_{3} C^{*}+(1-k)^{2} \lambda_{3} \varepsilon}-1\right)
\end{gathered}
$$

, otherwise unstable.

Proof : We linearised system (5) around the Type 2-Dead free equilibrium point $P_{d 2}$, we obtained the following Jacobian matrix $J\left(P_{d 2}\right)$ at $P_{d 2}=\left(0, \frac{d \alpha_{2}-\gamma_{2} m_{1}^{*}-\mu_{5}}{\mu_{2}}, m_{1}^{*}, \frac{(1-k) \varepsilon}{\mu_{4}}\right)$

$$
J=\left(\begin{array}{cccc}
\alpha_{1}-(1-k) \lambda_{1} E_{1}^{*} & 0 & 0 & 0 \\
(1-k) \lambda_{1} E_{1}^{*} & Q_{1} & -\gamma_{2} T_{1}^{*} & 0 \\
0 & Q_{2} & Q_{3} & \frac{\lambda_{3} g M_{1}^{*}(1-k)}{\left(g+E_{1}^{*}\right)^{2}} \\
0 & 0 & 0 & -\mu_{4}
\end{array}\right)
$$

where,

$$
Q_{1}=\left(d \alpha_{2}-2 \mu_{2} T_{1}^{*}-\gamma_{2} M_{1}^{*}-\mu_{5}\right), Q_{2}=\left(\frac{\rho \omega \mu_{2}^{2} M_{1}^{*}-\gamma_{3} M_{1}^{*}\left(\omega \mu_{2}+d \alpha_{2}-\gamma_{2} M_{1}^{*}-\mu_{5}\right)^{2}}{\left(\omega \mu_{2}+d \alpha_{2}-\gamma_{2} M_{1}^{*}-\mu_{5}\right)^{2}}\right), Q_{3}=\left(\frac{\rho T_{1}^{*}}{\omega+T_{1}^{*}}-\gamma_{3} T_{1}^{*}-\mu_{3}-\frac{(1-k) \lambda_{3} E_{1}^{*}}{g+E_{1}^{*}}\right)
$$




$$
\begin{gathered}
\left|J\left(P_{2}\right)\right|=\left(\begin{array}{cccc}
B_{0} & 0 & 0 & 0 \\
B_{1} & B_{2} & -B_{4} & 0 \\
0 & B_{3} & B_{5} & B_{6} \\
0 & 0 & 0 & -\mu_{4}
\end{array}\right) \\
\left|J\left(P_{2}\right)\right|=\left|\begin{array}{cccc}
B_{0}-\delta & 0 & 0 & 0 \\
B_{1} & B_{2}-\delta & -B_{4} & 0 \\
0 & B_{3} & B_{5}-\delta & B_{6} \\
0 & 0 & 0 & -\mu_{4}-\delta
\end{array}\right|=0
\end{gathered}
$$

Clearly, one of the eigenvalues of the system (5) at $\left|J\left(P_{2}\right)\right|$ is negative and real i.e. $\delta_{1}=-\mu_{4}$. However, the remaining can be analysed by simple calculation.

$$
\begin{aligned}
& \left(B_{0}-\delta\right)\left(B_{2}-\delta\right)\left(B_{5}-\delta\right)=0 \\
& \Rightarrow B_{5}=\delta_{2}, B_{2}=\delta_{3}, B_{0}=\delta_{4}
\end{aligned}
$$

Where ;

$$
\begin{gathered}
B_{0}=\frac{\alpha_{1} \mu_{4}-(1-k)^{2} \lambda_{1} \varepsilon}{\mu_{4}}, B_{2}=\gamma_{2} M_{1}^{*}-\alpha_{2} d+\mu_{5} \\
B_{5}=\frac{\left(A^{*} \rho \mu_{2}^{2}-A^{*} C^{*} \gamma_{3}\right)\left(d \alpha_{2}-\gamma_{2} M_{1}^{*}-\mu_{5}\right)-C^{*} \mu_{2}\left(\mu_{3} A^{*}+(1-k)^{2} \lambda_{3} \varepsilon\right)}{\mu_{2} A^{*} C^{*}}
\end{gathered}
$$

where: $A^{*}=\left(g \mu_{4}-(1-k) \varepsilon\right) \quad$ and, $C^{*}=\left(\omega \mu_{2}+d \alpha_{2}-\gamma_{2} M_{1}^{*}-\mu_{5}\right)$

It follows the following conditions

i) $B_{0}<0$ if , $\alpha_{1} \leq 1,0 \leq k<1$ and $\left(\frac{(1-k)^{2} \lambda_{1} \varepsilon}{\alpha_{1} \mu_{4}}\right)>1$

ii)

$$
B_{5}<0 \quad \text { provided }
$$$$
A^{*}>0
$$$$
0 \leq k \leq 1
$$

and $\omega>\frac{A^{*}}{\mu_{2}}\left(\frac{\mu_{2} C^{*} \rho}{\gamma_{3} A^{*} C^{*}+\mu_{2} \mu_{3} C^{*}+(1-k)^{2} \lambda_{3} \varepsilon}-1\right)$

\subsection{Co-existing equilibrium points}

Theorem 5: The co-existing equilibrium point $P_{e}$ of system (5) is locally asymptotically stable if

$$
\left(B_{0} B_{3}+B_{0} B_{6}+B_{3} B_{6}+B_{4} B_{5}\right)>B_{1} B_{2}
$$

, otherwise unstable.

Proof : We analysed and linearised system (5) around the Co-existing equilibrium point $P_{e}$, we obtained the following Jacobian matrix $J\left(P_{e}\right)$ at $P_{e}=\left(N_{4}^{*}, T_{4}^{*}, M_{4}^{*}, E_{4}^{*}\right)$

Co-existing equilibrium state exists when all cells population would have survived the competition. where $N_{4}^{*}, T_{4}^{*}, M_{4}^{*} \& E_{4}^{*}$ represent the coexisting equilibrium values for normal 
cells, tumor cells, immune cells and estrogen levels respectively.

$$
N_{4}^{*}=\frac{2(1-k)^{4} \lambda_{1}^{4} \mu_{1} \mu_{4} \varepsilon^{2}+\Phi_{1} \alpha_{1}^{2} \mu_{4}^{2} \mu_{1}-2(1-k)^{2} \mu_{1} \mu_{4}^{2} \alpha_{1} \lambda_{1} \Phi_{1} \varepsilon-2 \alpha_{1} \Phi_{1}^{2} \mu_{1} \mu_{4}^{3}-2(1-k)^{2} \alpha_{1} \mu_{1} \mu_{4}^{2} \lambda_{1} \varepsilon}{2 \Phi_{1} \alpha_{1} \mu_{1}^{2} \mu_{4}^{3}-2(1-k)^{2} \mu_{1}^{2} \mu_{4}^{2} \lambda_{1} \Phi_{1} \varepsilon}
$$

$$
T_{4}^{*}=\frac{\alpha_{1}^{2} \mu_{1} \mu_{4}^{2}+2 \alpha_{1} \mu_{1} \mu_{4}^{2} \Phi_{1}}{2 \Phi_{1} \alpha_{1} \mu_{1} \mu_{4}^{2}-2(1-k)^{2} \mu_{1} \mu_{4} \lambda_{1} \Phi_{1} \varepsilon}
$$

$M_{4}^{*}=\frac{G^{2} Z^{*}(1-k)^{2} \lambda_{1} \varepsilon+\left(\alpha_{1}^{2} \alpha_{2} \mu_{1} \mu_{4}^{3} d+2 \alpha_{1} \alpha_{2} \mu_{4} \mu_{4}^{3} \Phi_{1} d-\mu_{4}^{3} \mu_{5} \alpha_{1}^{2}-2 \mu_{4}^{3} \mu_{1} \mu_{5} \alpha_{1} \Phi_{1}\right) G^{*}-\mu_{3} \alpha_{1}^{4} \mu_{1}^{2} \mu_{4}^{5}-4 \alpha_{1}^{2} \mu_{1}^{2} \mu_{2} \mu_{4}^{5} \Phi_{1}-4 \Phi_{1}^{2} \alpha_{1}^{2} \mu_{1}^{2} \mu_{4}^{5} \mu_{2}}{G^{*} Q^{*} \mu_{4}}$ $E_{4}^{*}=\frac{(1-k) \mathcal{\varepsilon}}{\mu_{4}}$

Where;

$$
G^{*^{2}}=2 \Phi_{1} \alpha_{1} \mu_{1} \mu_{4}^{2}-2(1-k)^{2} \mu_{1} \mu_{4} \lambda_{1} \Phi_{1} \varepsilon
$$

$$
Z^{*}=\frac{2(1-k)^{4} \lambda_{1}^{2} \mu_{1} \mu_{4} \varepsilon^{2}+\Phi_{1} \alpha_{1}^{2} \mu_{4}^{2} \mu_{1}-2(1-k)^{2} \mu_{1} \mu_{4}^{2} \alpha_{1} \lambda_{1} \Phi_{1} \varepsilon-2 \alpha_{1} \Phi_{1}^{2} \mu_{1} \mu_{4}^{3}-2(1-k)^{2} \alpha_{1} \mu_{1} \mu_{4}^{2} \lambda_{1} \varepsilon}{2 \Phi_{1} \alpha_{1} \mu_{1}^{2} \mu_{4}^{3}-2(1-k)^{2} \mu_{1}^{2} \mu_{4}^{2} \lambda_{1} \Phi_{1} \varepsilon}
$$

$$
\begin{aligned}
& Q^{*}=\frac{\alpha_{1}^{2} \mu_{1} \mu_{4}^{2} \gamma_{2}-2 \alpha_{1} \mu_{1} \mu_{4}^{2} \Phi_{1} \gamma_{2}}{2 \Phi_{1} \alpha 1 \mu_{1} \mu_{4}^{2}-2(1-k)^{2} \mu_{1} \mu_{4} \lambda_{1} \Phi_{1} \varepsilon} \\
& J=\left(\begin{array}{cccc}
\left(\alpha_{1}-2 \mu_{1} N_{4}^{*}-(1-k) \lambda_{1} E_{4}^{*}\right) & -N_{4}^{*} \phi & 0 & -B_{7} \\
(1-k) \lambda_{1} E_{4}^{*} & \left(d \alpha_{2}-2 \mu_{2} T_{4}^{*}-\gamma_{2} M_{4}^{*}-\mu_{5}\right) & -\gamma_{2} T_{4}^{*} & B_{7} \\
0 & B_{4} & B_{6} & \frac{\lambda_{3} g M_{4}^{*}(1-k)}{\left(g+E_{4}^{*}\right)^{2}} \\
0 & 0 & 0 & -\mu_{4}
\end{array}\right) \\
& J\left(P_{e}\right)=\left(\begin{array}{cccc}
B_{0} & -B_{2} & 0 & -B_{7} \\
B_{1} & B_{3} & -B_{5} & B_{7} \\
0 & B_{4} & B_{6} & B_{8} \\
0 & 0 & 0 & -\mu_{4}
\end{array}\right)
\end{aligned}
$$




$$
\left|J\left(P_{e}\right)\right|=\left|\begin{array}{cccc}
B_{0}-\delta & -B_{2} & 0 & -B_{7} \\
B_{1} & B_{3}-\delta & -B_{5} & B_{7} \\
0 & B_{4} & B_{6}-\delta & B_{8} \\
0 & 0 & 0 & -\mu_{4}-\delta
\end{array}\right|=0
$$

Obviously, $\delta_{1}=-\mu_{4}$ is negative and real. However, the remaining three eigenvalues formed

$$
\delta^{3}-\left(B_{0}+B_{3}+B_{6}\right) \delta^{2}+\left(B_{0} B_{3}+B_{0} B_{6}+B_{3} B_{6}+B_{4} B_{5}+B_{1} B_{2}\right) \delta-\left(B_{0} B_{3} B_{6}+B_{0} B_{4} B_{5}+B_{1} B_{2} B_{6}\right)=0
$$

where;

$$
\begin{gathered}
B_{0}=\frac{\alpha_{1} \mu_{4}-2 \mu_{1} \mu_{4} N_{4}^{*}-(1-k)^{2} \lambda_{1} \varepsilon}{\mu_{4}}, B_{1}=\frac{(1-k)^{2} \lambda_{1} \varepsilon}{\mu_{4}}, B_{2}=-\phi_{1} N_{4}^{*}, \\
B_{3}=\left(d \alpha_{2}-2 \mu_{2} T_{4}^{*}-\gamma_{2} M_{4}^{*}-\mu_{5}\right), B_{4}=\frac{\rho M_{4}^{*} \omega-\gamma_{3} M_{4}^{*}\left(\omega+T_{4}^{*}\right)^{2}}{\left(\omega+T_{4}^{*}\right)^{2}} B_{5}=-\gamma_{2} T_{4}^{*} \\
B_{6}=\frac{\rho T_{4}^{*}\left(g \mu_{4}+(1-k) \varepsilon\right)-\gamma_{3} T_{4}^{*}\left(\omega+T_{4}^{*}\right)\left(g \mu_{4}+(1-k) \varepsilon\right)-\mu_{3}\left(\omega+T_{4}^{*}\right)\left(g \mu_{4}+(1-k) \varepsilon\right)-(1-k)^{2}\left(\omega+T_{4}^{*}\right) \lambda_{3} \varepsilon}{\left(\omega+T_{4}^{*}\right)\left(g \mu_{4}+(1-k) \varepsilon\right)} \\
-B_{7}=-(1-k) \lambda_{1} N_{4}^{*}, B_{7}=(1-k) \lambda_{1} N_{4}^{*}, B_{8}=\frac{\lambda_{3} \mu_{4}^{2} g M_{4}^{*}(1-k)}{\left(g \mu_{4}+(1-k) \varepsilon\right)^{2}}
\end{gathered}
$$

By Descartes' rule of sign it implies that equation (39) we have no sign changes if $B_{0}<0$, $B_{3}<0, \quad B_{6}<0, \quad B_{4}<0, \quad B_{6}<0 \quad$ and $\quad\left(B_{0} B_{3}+B_{0} B_{6}+B_{3} B_{6}+B_{4} B_{5}\right)>B_{1} B_{2}$ Hence, all eigenvalues $\delta_{2}, \delta_{3}$, and $\delta_{4}$ are real negative. Therefore, co-existing equilibrium is locally asymptotically stable otherwise unstable.

since, $\varepsilon, k, \mu_{4}$ are positive parameters, we have $E_{4}^{*} \geq 0$;

$$
\alpha_{1} \geq \frac{2(1-k)^{2} \mu_{1}^{3} \mu_{4}^{5} \lambda_{1} \Phi_{1} \varepsilon \alpha_{1}}{2 \Phi_{1} \alpha_{1} \mu_{1} \mu_{4}}
$$

for $N_{4}^{*}$ to be feasible at this equilibrium state. However, the rate of normal cell growth must be greater than the rate at which they are lost as a result of interactions with tumor, the presence of more estrogen. The value of $T_{4}^{*}>0$ at the $P_{e}$ when $G \geq 0$.

\section{$4 \quad$ Uncertainty and sensitivity analysis}

In this section, we explore the dependence of the model solutions on the parameter values. We are able to figure-out a feasible range of parameter values and determine the most 
critical parameters in the model. We employ similar method which are discussed in detail by $[11,22]$, that is Latin Hypercube Sampling (LHS) for studying uncertainty analysis and Partial Rank Correlation Coefficient (PRCC) for analysing the sensitivity analysis indexes of the parameters. LHS/PRCC was ran and analysed with a sample size of 100 . The choice of this sample size is due to the fact that PRCC produces accurate results for a lower sample size compared to other technique like eFAST [11].

Uncertainty and sensitivity analysis were performed on all non-dimensional system parameters in the system (5) with the aim of determining the most sensitive parameters to the model. The parameter baseline values in Table 1 were varied in the range of $25 \%$. Figure 1.0 displays a bar graph of PRCCs plotted against the homogeneous parameter value with tumor compartment as the baseline dependent variable. The parameter which are significantly positively correlated with tumor cells, at $P<0.05$ level of significance, are $\alpha_{1}, g$ while $\mu_{1}, \gamma_{3}$, and $\omega$ is significantly negatively correlated. An increase in the production of normal cells $\alpha_{1}$, leads to higher numbers of normal cells, thus the higher the $\alpha_{1}$ the higher the normal cells

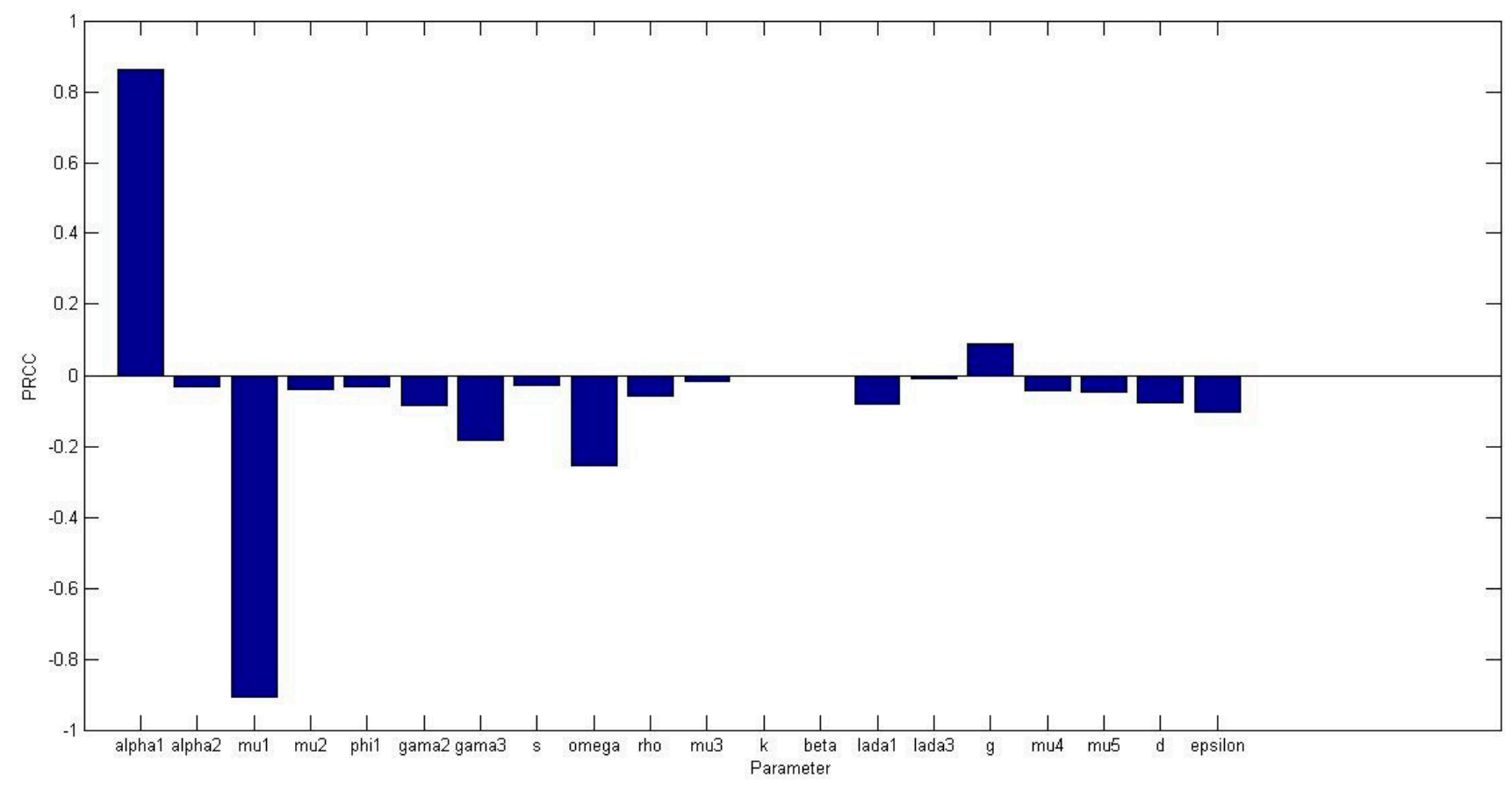

Figure 1: PRCCs of homogeneous model parameters with the tumor cells as the baseline variable. All parameter values were varied in $25 \%$ of their baseline values in Table 1. The most sensitive parameters are shown to be $P$-values of $\alpha_{1}, g, \mu_{1}, \gamma_{3}$ and $\omega$ are less than 0.01 . 


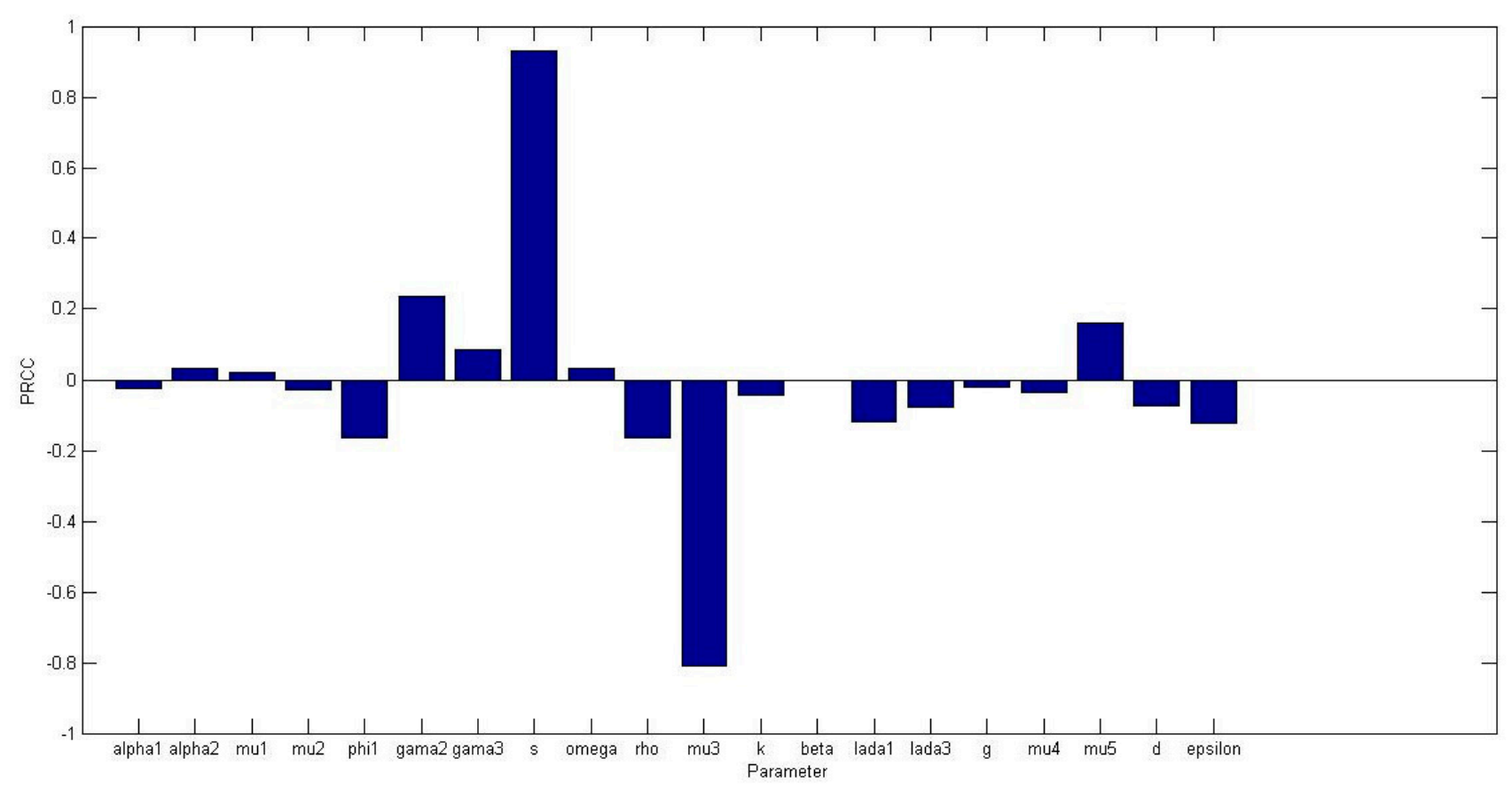

Figure 2: PRCCs of homogeneous model parameters with the tumor cells as the baseline variable. All parameter values were varied in $25 \%$ of their baseline values in Table 1. The most sensitive parameters are shown to be $P$-values of $s, \gamma_{2}, \mu_{3}$ and $\rho$ are less than 0.01

\section{Optimal control}

In the previous section, the controls on disease (ketogenic diet, immune booster and anti-cancer treatment) are considered as constants and hence no cost determination is taken care of which will be incurred in their implementation. In this section, we formulate a corresponding optimal control problem for the model in system (5) considering the ketogenic diet and chemotherapy as control interventions to minimize the cancer prevalence and corresponding economic burden. Optimal control technique has been used successfully to determine the relevant control strategies with optimal cost [9]

We use a quadratic term for rate of application of a anti-cancer drug control. Our goal is to minimize the cost and tumor cells growth while maximizing the tumor-free population. Mathematically, the objective functional $J_{1}$ formulates the optimization problem of interest, namely that of identifying the most effective strategies. The overall pre-selected objective involves the minimization of the number of cancer cells and treatment of individuals at a minimal cost over a finite time interval $\left\lfloor 0, T_{f}\right\rfloor[29,30,31]$. Then, we formulate the objective functional $J_{1}$

$$
J_{1}\left(u_{1}, u_{2}\right)=\int_{0}^{T} f\left(A_{1} T(t)+A_{2} E(t)+\frac{1}{2} A_{3} u_{1}^{2}(t)+\frac{1}{2} A_{4} u_{2}^{2}(t)\right) d t
$$

We choose (as it is customary) to model the control efforts through a linear combination of 
quadratic terms, $u_{i}^{2}(t)$ where $(i=1,2)$. The constants $A_{1}, A_{2}, A_{3}$ and $A_{4}$ are a measure of the relative cost of the interventions over $[0, T]$. The optimal control problem is that of finding optimal functions $\left(u_{1}^{*}(t), u_{2}^{*}(t)\right)$ such that

$$
J_{1}\left(u_{1}^{*}(t), u_{2}^{*}(t)\right)=\min _{\Omega} J_{1}\left(u_{1}(t), u_{2}(t)\right)
$$

where

$$
\Omega=\left\{u_{1}(t) \& u_{2}(t): 0 \leq u_{1}(t) \leq u_{1 \max }, 0 \leq u_{2}(t) \leq u_{2 \max }, t \in\left[0, T_{f}\right]\right\}
$$

Three different control strategies are explored. This approach can be used to test various options. However, we only look at the following three alternatives:

- Strategy 1: Anti-cancer drug treatment control on tumor cells (control $u_{1}(t)$

only)

- Strategy 2: Ketogenic diet control on excess estrogen and tumor cells (control $u_{2}(t)$ only)

- Strategy 3: Anti-cancer drug and ketogenic diet treatment combined control on tumor cells growth and excess estrogen (controls $u_{1}(t)$ and $u_{2}(t)$ )

Thus, strategies 1-3 use the objective functionals (52)-(54), respectively.

$$
\begin{gathered}
J_{1}\left(u_{1}(t)\right)=\int_{0}^{T_{f}}\left(T(t)+\frac{1}{2} A_{3} u_{1}^{2}(t)\right) d t \\
J_{1}\left(u_{2}(t)\right)=\int_{0}^{T_{f}}\left(E(t)+\frac{1}{2} A_{4} u_{2}^{2}(t)\right) d t \\
J_{1}\left(u_{1}, u_{2}\right)=\int_{0}^{T_{f}}\left(A_{1} T(t)+A_{2} E(t)+\frac{1}{2} A_{3} u_{1}^{2}(t)+\frac{1}{2} A_{4} u_{2}^{2}(t)\right) d t
\end{gathered}
$$

We assume there are practical limitations on the maximum rate at which the anti-cancer treatment may be applied in a given time period and we define the positive constant $u_{\max }$ accordingly. We also define the set $\Omega$ of admissible controls to be all Lebesgue measurable functions which take on values in the control set [30].

$u=\left[0, u_{\max }\right]$ almost everywhere on $[0, T]$ we seek an optimal control $u^{*} \in \Omega$ in (51)[29].

Equation (50) is subject to:

$$
\begin{aligned}
& \frac{d N}{d t}=N \alpha_{1}-\mu_{1} N^{2}-\phi_{1} T N-\left(1-u_{1}(t)\right)\left(\lambda_{1} N E\right) \\
& \frac{d T}{d t}=\left(1-u_{2}(t)\right) T \alpha_{2}-\mu_{2} T^{2}-\gamma_{2} M T-\mu_{5} T+\left(1-u_{1}(t)\right)\left(\lambda_{1} N E\right) \\
& \frac{d M}{d t}=s \beta+\frac{\rho M T}{\omega+T}-\gamma_{3} M T-\mu_{3} M-\left(\left(1-u_{1}(t)\right) \frac{\lambda_{3} M E}{g+E}\right) \\
& \frac{d E}{d t}=\left(1-u_{1}(t)\right) \varepsilon-\mu_{4} E
\end{aligned}
$$

For the purpose of necessary conditions for optimal control functions with the help of 
Pontryagin's Maximum Principle [14] for the system (50) - (52)

$$
H\left(N, T, M, E, u_{1}, u_{2}, \theta_{i}\right)=L\left(N, T, M, E, u_{1}, u_{2}, \theta_{i}\right)+\theta_{1} N^{\prime}+\theta_{2} T^{\prime}+\theta_{3} M^{\prime}+\theta_{4} E^{\prime}
$$

where; $L$ is the Lagrangian function,

$$
L\left(N, T, M, E, u_{1}, u_{2}\right)=A_{1} T(t)+A_{2} E(t)+\frac{1}{2} A_{3} u_{1}^{2}(t)+\frac{1}{2} A_{4} u_{2}^{2}(t) \geq \frac{1}{2} A_{3} u_{1}^{2}(t)+\frac{1}{2} A_{4} u_{2}^{2}(t)
$$

$$
H=\left\{\begin{array}{c}
A_{1} T(t)+A_{2} E(t)+\frac{1}{2} A_{3} u_{1}^{2}(t)+\frac{1}{2} A_{4} u_{2}^{2}(t) \\
+\theta_{1}\left(N \alpha_{1}-\mu_{1} N^{2}-\phi_{1} T N-\left(1-u_{1}(t)\right)\left(\lambda_{1} N E\right)\right) \\
+\theta_{2}\left(\left(1-u_{2}(t)\right) T \alpha_{2}-\mu_{2} T^{2}-\gamma_{2} M T-\mu_{5} T+\left(1-u_{1}(t)\right)\left(\lambda_{1} N E\right)\right) \\
+\theta_{3}\left(\begin{array}{c}
\left.s \beta+\frac{\rho M T}{\omega+T}-\gamma_{3} M T-\mu_{3} M-\left(\left(1-u_{1}(t)\right) \frac{\lambda_{3} M E}{g+E}\right)\right) \\
+\theta_{4}\left(\left(1-u_{1}(t)\right) \varepsilon-\mu_{4} E\right)
\end{array}\right.
\end{array}\right.
$$

where $\theta_{1}, \theta_{2}, \theta_{3}, \theta_{4}$

are adjoints variable for the states N,T,M,E. However, with the help of Pontryagin's Maximum Principle, we can obtain minimised Hamiltonian that minimizes objective function or cost functional.

We apply Pontryagin's Maximum Principle [14], to characterize the optimal control pair $u_{1}^{*} \& u_{2}^{*}$ in the following result.

Theorem 5.1: Given optimal control variables $u_{1}^{*} \& u_{2}^{*}$ and $N^{*}, T^{*}, M^{*} \& E^{*}$ are corresponding optimal state variables of the control system (50) and (52). Then there exists adjoint variable

$$
\begin{aligned}
& \theta=\left(\theta_{1}, \theta_{2}, \theta_{3}, \theta_{4}\right) \in \mathfrak{R}_{+}^{4} \text { that satisfies the following equations. } \\
& \frac{d \theta_{1}}{d t}=2 \theta_{1} \mu_{1} N+\phi_{1} \theta_{1} T+\left(\theta_{1}+\theta_{2}\right)\left(1-u_{2}(t)\right) \lambda_{1} E-\alpha_{1} \theta_{1} \\
& \frac{d \theta_{2}}{d t}=-A_{1}+\theta_{1} \phi_{1} N+\theta_{2}\left(2 T \mu_{2}+\gamma_{2} M+\mu_{5}-\alpha_{2}\left(1-u_{2}\right)\right)+\theta_{3}\left(\gamma_{3} M-\frac{\rho \omega M}{(\omega+T)^{2}}\right) \\
& \frac{d \theta_{3}}{d t}=\theta_{2} \gamma_{2} T-\rho \theta_{3} T+\gamma_{3} \theta_{3} T+\mu_{3} \theta_{3}+\theta_{3}\left(\left(1-u_{1}\right) \frac{\lambda_{1} E}{g+E}\right) \\
& \frac{d \theta_{4}}{d t}=-A_{2}+\left(\theta_{1}-\theta_{2}\right)\left(1-u_{1}\right) \lambda_{1} N-\theta_{3}\left(\left(1-u_{1}\right) \frac{\lambda_{3} M g}{(g+E)^{2}}\right)-\theta_{4} \mu_{4}
\end{aligned}
$$

with transversality conditions

$\theta_{1}\left(T_{f}\right)=\theta_{2}\left(T_{f}\right)=\theta_{3}\left(T_{f}\right)=\theta_{4}\left(T_{f}\right)=0$

The corresponding optimal controls $u_{1}^{*} \& u_{2}^{*}$ are given as, 


$$
u_{1}^{*}=\min \left\{\max \left\{0, \frac{1}{A_{3}}\left(\theta_{2} \lambda_{1} N^{*} E^{*}+\theta_{3} \varepsilon-\theta_{1} \lambda_{1} N^{*} E^{*}-\frac{\theta_{3} \lambda_{3} M^{*} E^{*}}{g+E^{*}}\right)\right\}, 1\right\}
$$

and

$$
u_{2}^{*}=\min \left\{\max \left\{0, \frac{1}{A_{4}}\left(\theta_{2} \alpha_{2} T^{*}\right)\right\}, 1\right\}
$$

Proof: Let $u_{1}^{*} \& u_{2}^{*}$ be the given optimal control functions and $N^{*}, T^{*}, M^{*} \& E^{*}$ be the corresponding optimal state variables of the system (7) which minimize the cost functional or objective (6). Then by Pontryagin's Maximum Principle [14], there exist adjoint variables $\theta_{1}, \theta_{2}, \theta_{3}, \& \theta_{4}$ which satisfy following equations:

$$
\frac{d \theta_{1}}{d t}=-\frac{\partial H}{\partial N}, \frac{d \theta_{2}}{d t}=-\frac{\partial H}{\partial T}, \frac{d \theta_{3}}{d t}=-\frac{\partial H}{\partial M}, \frac{d \theta_{4}}{d t}=-\frac{\partial H}{\partial E}
$$

with transversality conditions

$$
\theta_{1}\left(T_{f}\right)=\theta_{2}\left(T_{f}\right)=\theta_{3}\left(T_{f}\right)=\theta_{4}\left(T_{f}\right)=0
$$

where $\mathrm{H}$ is the Hamiltonian and defined as :

$$
H\left(N, T, M, E, u_{1}, u_{2}, \theta\right)=L\left(N, T, M, E, u_{1}, u_{2}\right)+\theta_{1} N^{\prime}+\theta_{2} T^{\prime}+\theta_{3} M^{\prime}+\theta_{4} E^{\prime}
$$

$$
H=\left\{\begin{array}{c}
A_{1} T(t)+A_{2} E(t)+\frac{1}{2} A_{3} u_{1}^{2}(t)+\frac{1}{2} A_{4} u_{2}^{2}(t) \\
+\theta_{1}\left(N \alpha_{1}-\mu_{1} N^{2}-\phi_{1} T N-\left(1-u_{1}(t)\right)\left(\lambda_{1} N E\right)\right) \\
+\theta_{2}\left(\left(1-u_{2}(t)\right) T \alpha_{2}-\mu_{2} T^{2}-\gamma_{2} M T-\mu_{5} T+\left(1-u_{1}(t)\right)\left(\lambda_{1} N E\right)\right) \\
+\theta_{3}\left(s \beta+\frac{\rho M T}{\omega+T}-\gamma_{3} M T-\mu_{3} M-\left(\left(1-u_{1}(t)\right) \frac{\lambda_{3} M E}{g+E}\right)\right) \\
+\theta_{4}\left(\left(1-u_{1}(t)\right) \varepsilon-\mu_{4} E\right)
\end{array}\right.
$$

from the optimality condition, we have

$$
\begin{gathered}
\frac{\partial H}{\partial u_{1}}=0, \text { at } u_{1}=u_{1}^{*} \text { and } \frac{\partial H}{\partial u_{2}}=0 \text {, at } u_{2}=u_{2}^{*} \text { which implies that, } \\
0=\frac{\partial H}{\partial u_{1}}=A_{3} u_{1}+\theta_{1} \lambda_{1} N E-\theta_{2} \lambda_{1} N E+\theta_{3} \frac{\lambda_{3} M E}{g+E}-\theta_{4} \varepsilon \\
0=\frac{\partial H}{\partial u_{1}}=A_{4} u_{2}-\theta_{2} \alpha_{2} T
\end{gathered}
$$

Hence, we obtain (see [10])

$$
u_{1}^{*}=\frac{1}{A_{3}}\left\{\theta_{1} \lambda_{1} N E+\theta_{4} \varepsilon-\theta_{1} \lambda_{1} N E-\theta_{3} \frac{\lambda_{3} M E}{g+E}\right\}
$$




$$
u_{2}^{*}=\frac{1}{A_{4}}\left\{\theta_{2} \alpha_{2} T\right\}
$$

Thus we have, (16) and (17).

By standard control arguments involving the bounds on the controls, we conclude

$$
u_{1}^{*}=\left\{\begin{array}{c}
0 \quad \text { if } \frac{1}{A_{3}}\left(\theta_{1} \lambda_{1} N E+\theta_{4} \varepsilon-\theta_{1} \lambda_{1} N E-\theta_{3} \frac{\lambda_{3} M E}{g+E}\right)<0 \\
\frac{1}{A_{3}}\left(\theta_{1} \lambda_{1} N E+\theta_{4} \varepsilon-\theta_{1} \lambda_{1} N E-\theta_{3} \frac{\lambda_{3} M E}{g+E}\right) \text { if } 0 \leq \frac{1}{A_{3}}\left(\theta_{1} \lambda_{1} N E+\theta_{4} \varepsilon-\theta_{1} \lambda_{1} N E-\theta_{3} \frac{\lambda_{3} M E}{g+E}\right) \leq 1 \\
1 \text { if } \frac{1}{A_{3}}\left(\theta_{1} \lambda_{1} N E+\theta_{4} \varepsilon-\theta_{1} \lambda_{1} N E-\theta_{3} \frac{\lambda_{3} M E}{g+E}\right)>1
\end{array}\right.
$$

and

$$
u_{2}^{*}=\left\{\begin{array}{ccc}
0 & \text { if } & \frac{1}{A_{4}}\left(\theta_{2} \alpha_{2} T^{*}\right)<0 \\
\frac{1}{A_{4}}\left(\theta_{2} \alpha_{2} T^{*}\right) & \text { if } 0 \leq \frac{1}{A_{4}}\left(\theta_{2} \alpha_{2} T^{*}\right) \leq 1 \\
1 & \text { if } & \frac{1}{A_{4}}\left(\theta_{2} \alpha_{2} T^{*}\right)>1
\end{array}\right.
$$

However, we discuss the numerical solution of the optimality system and the corresponding results of varying the optimal controls $u_{1} \& u_{2}$ the parameter choices, and the interpretations from various cases.

\section{Numerical Simulations and Discussion}

Numerical simulations of this model give us a pictures of the dynamical behaviour of breast cancer cells in the presence of normal cells, tumor cells, immune cells and estrogen. Maple 18 version was used for all our simulations for the system (5). Key parameters are also noted in stabilising the model in system (5) for example: ketogenic diet, anti-cancer and immune booster. The initial values of variables are $\mathrm{N}(0)=2000, \mathrm{~T}(0)=800, \mathrm{M}(0)=500, \mathrm{E}(0)=20$ and $s=1.3 \times 10^{4}$ adopted from [4]. All parameter values used for the numerical simulation are stated in Table 1 above. However, the numerical solutions generally show that in the presence of high level of estrogen the tumor cells proliferation rate increases in Fig.1 and it lead the system (5) unstable in Fig.9 


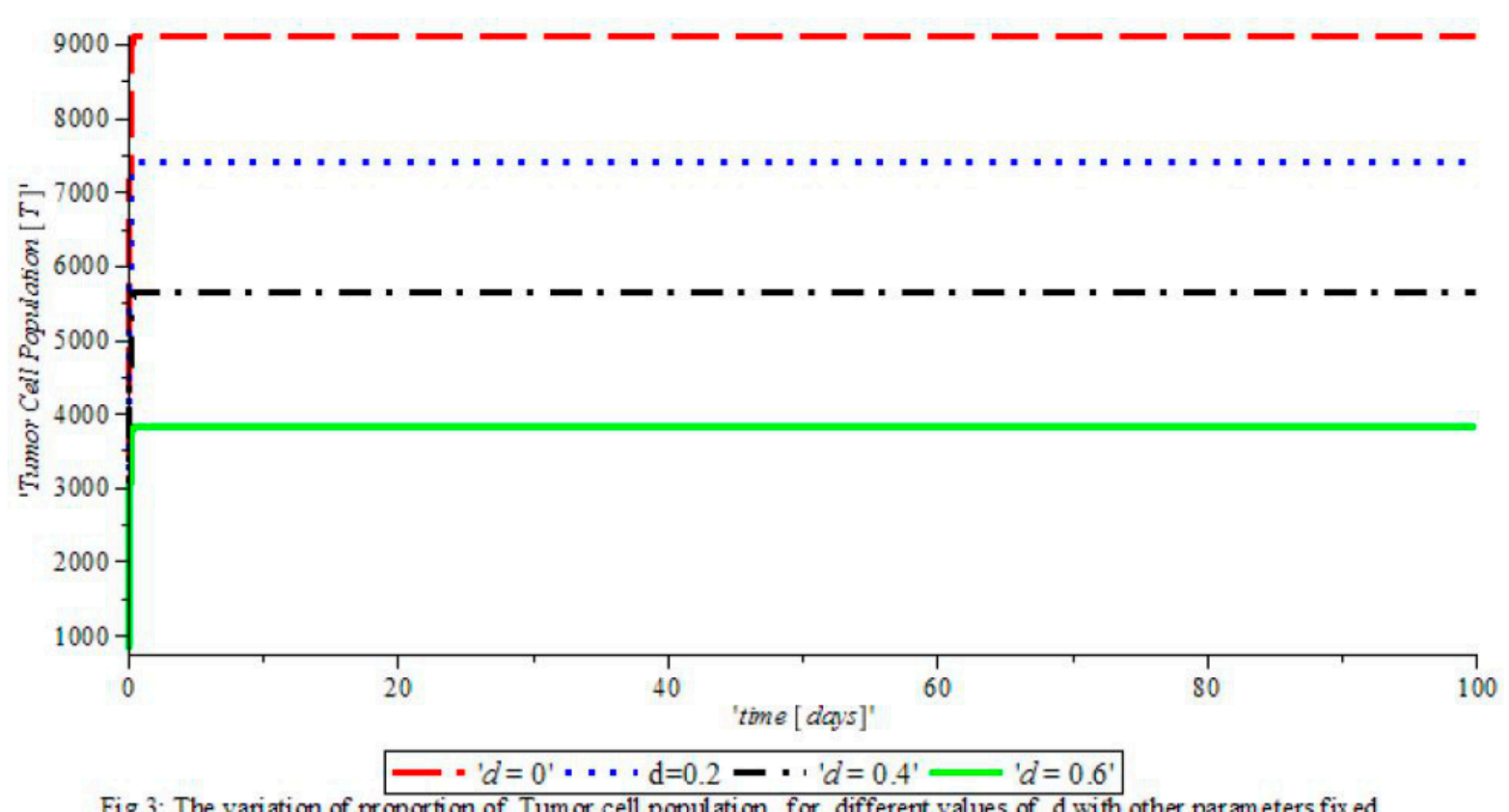

Fig 3: The variation of proportion of Tum or cell population for different values of $d$ with other parameters fix ed

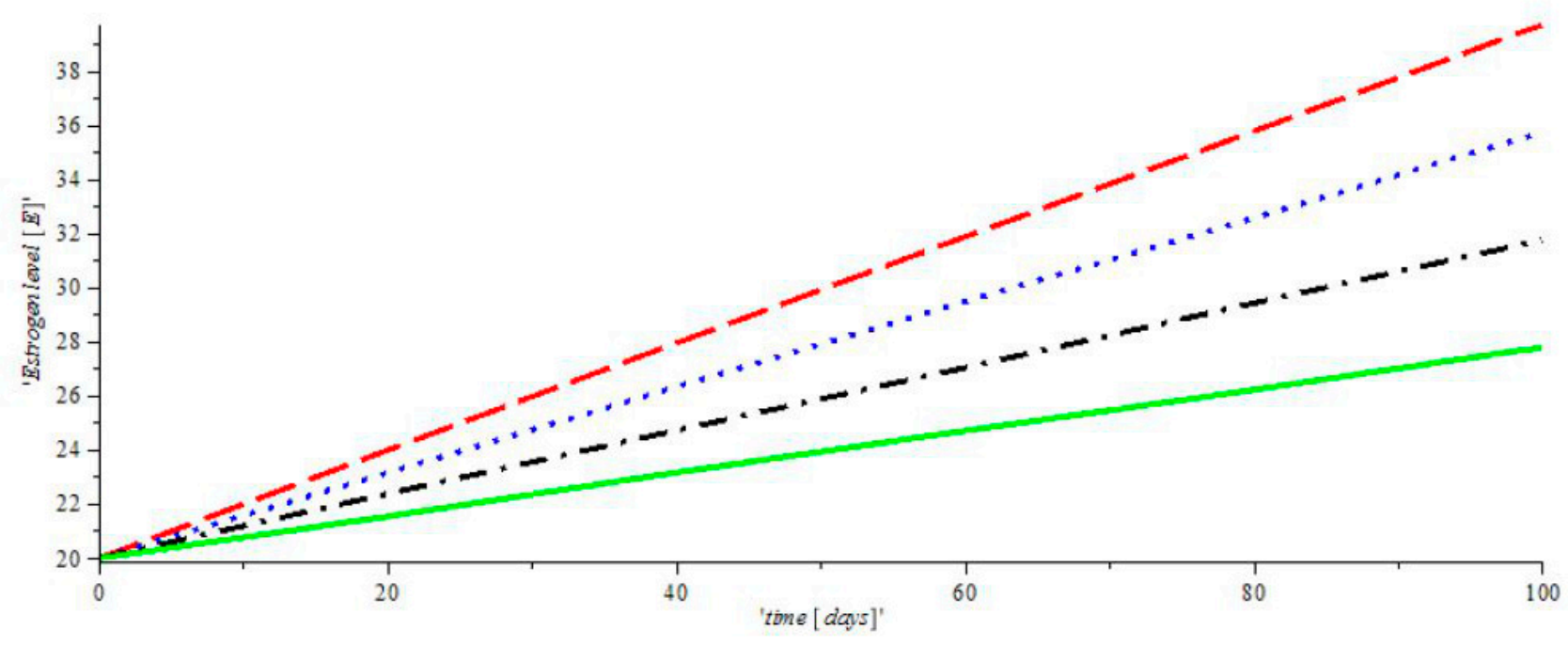

Fig 4: The variation of proportion of Estrogen level for different values of $\mathrm{k}$ with other parameters fixed 


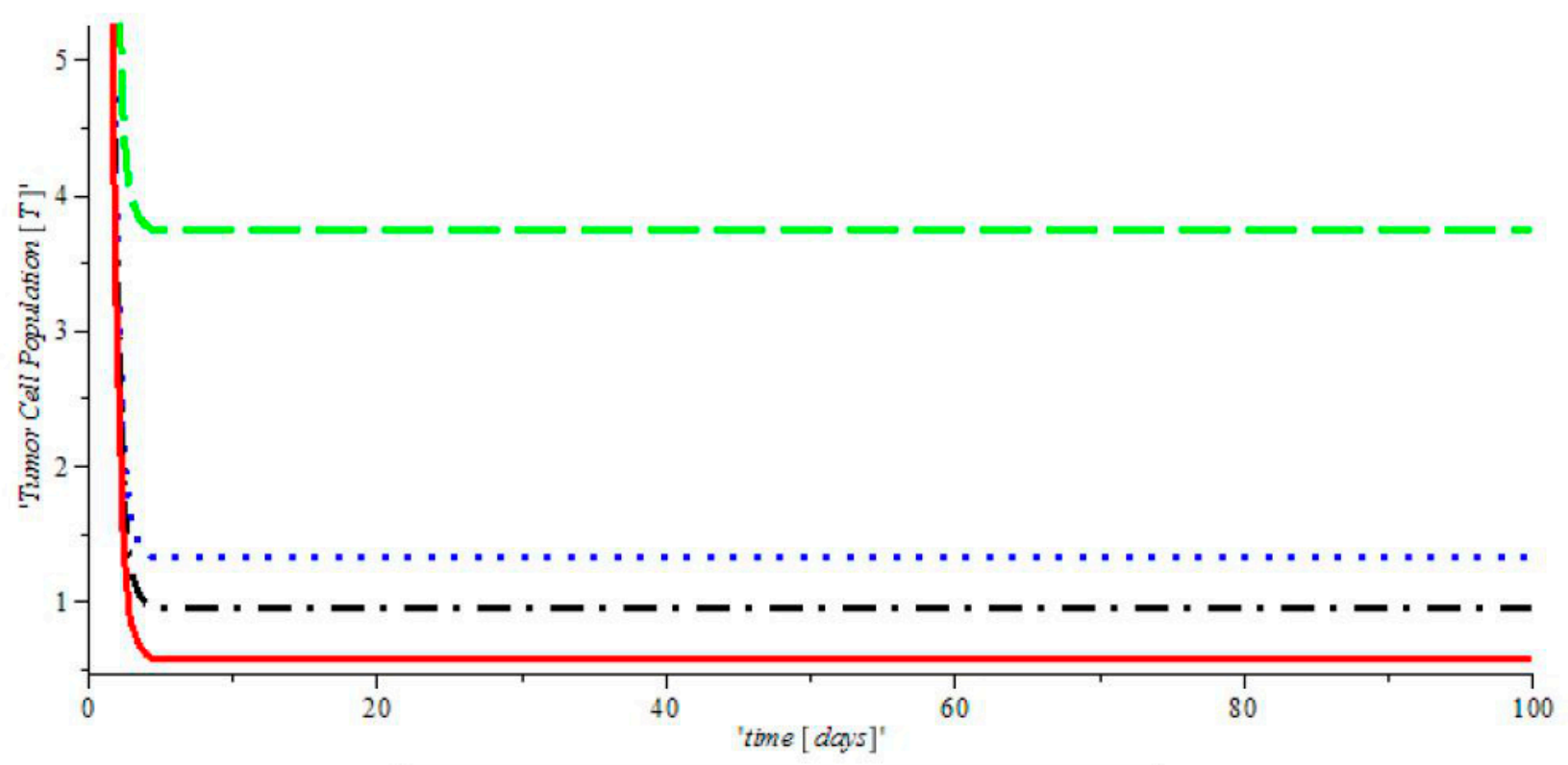

Fig 5: The variation of proportion of Tum or cells population for different values of $k$ with other parameters fix ed

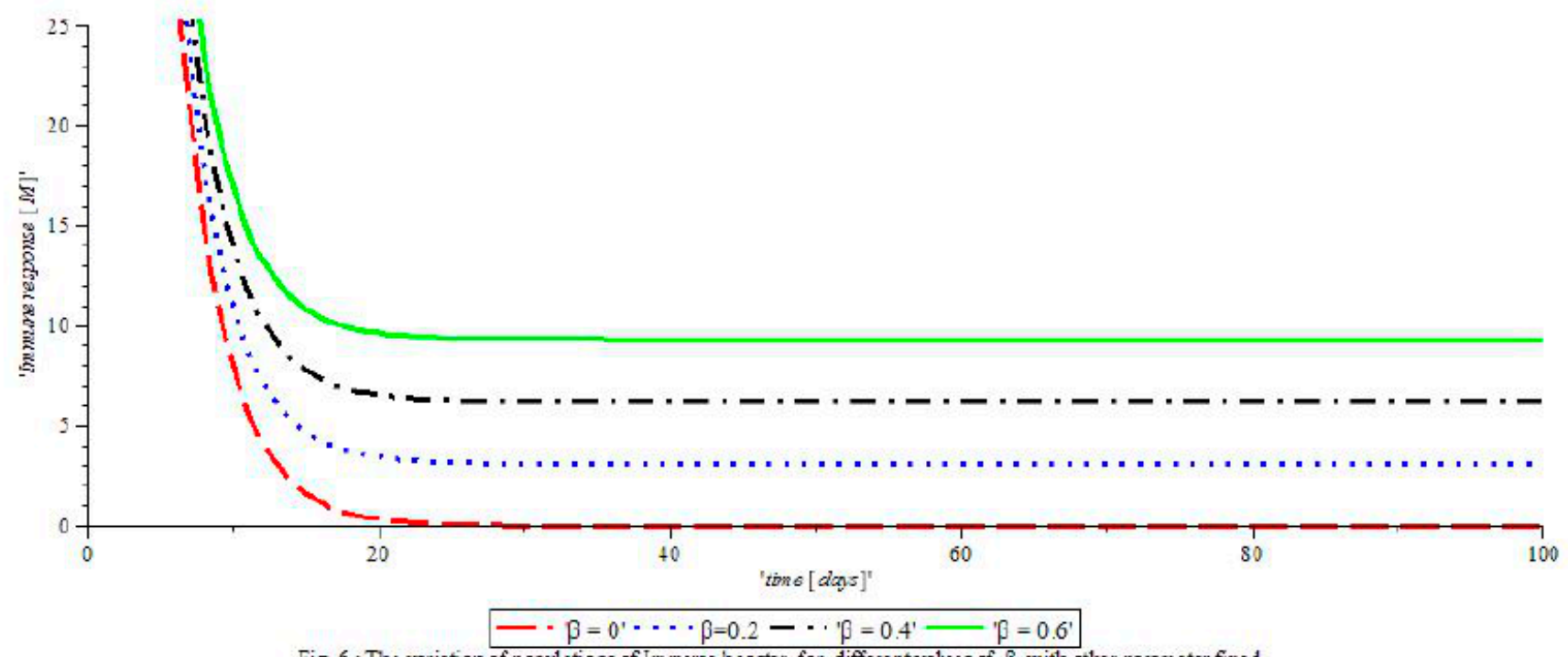

Fig 6 : The variation of populations of Imm une booster for differentvalues of $\beta$ with other parameter fixed 

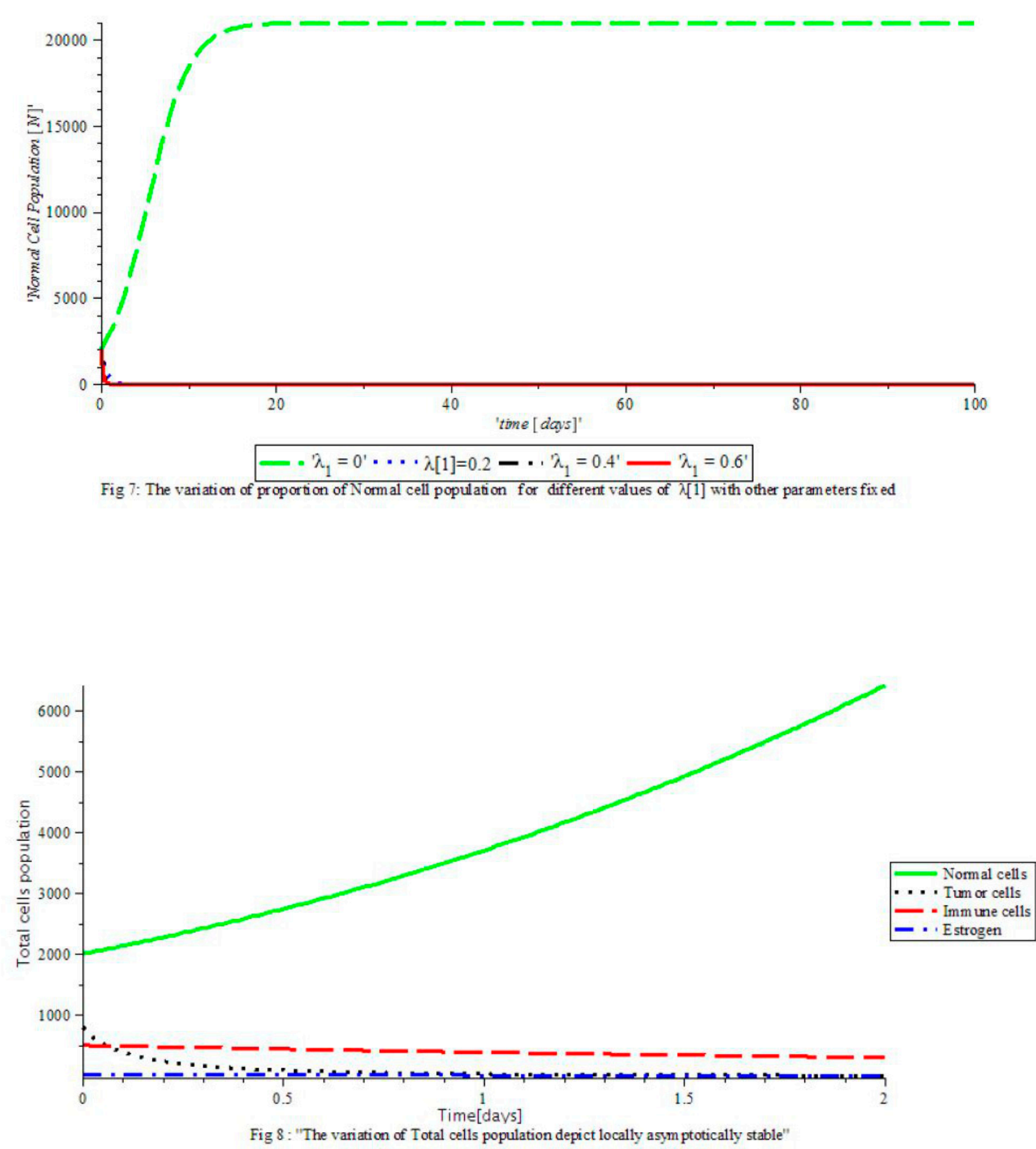


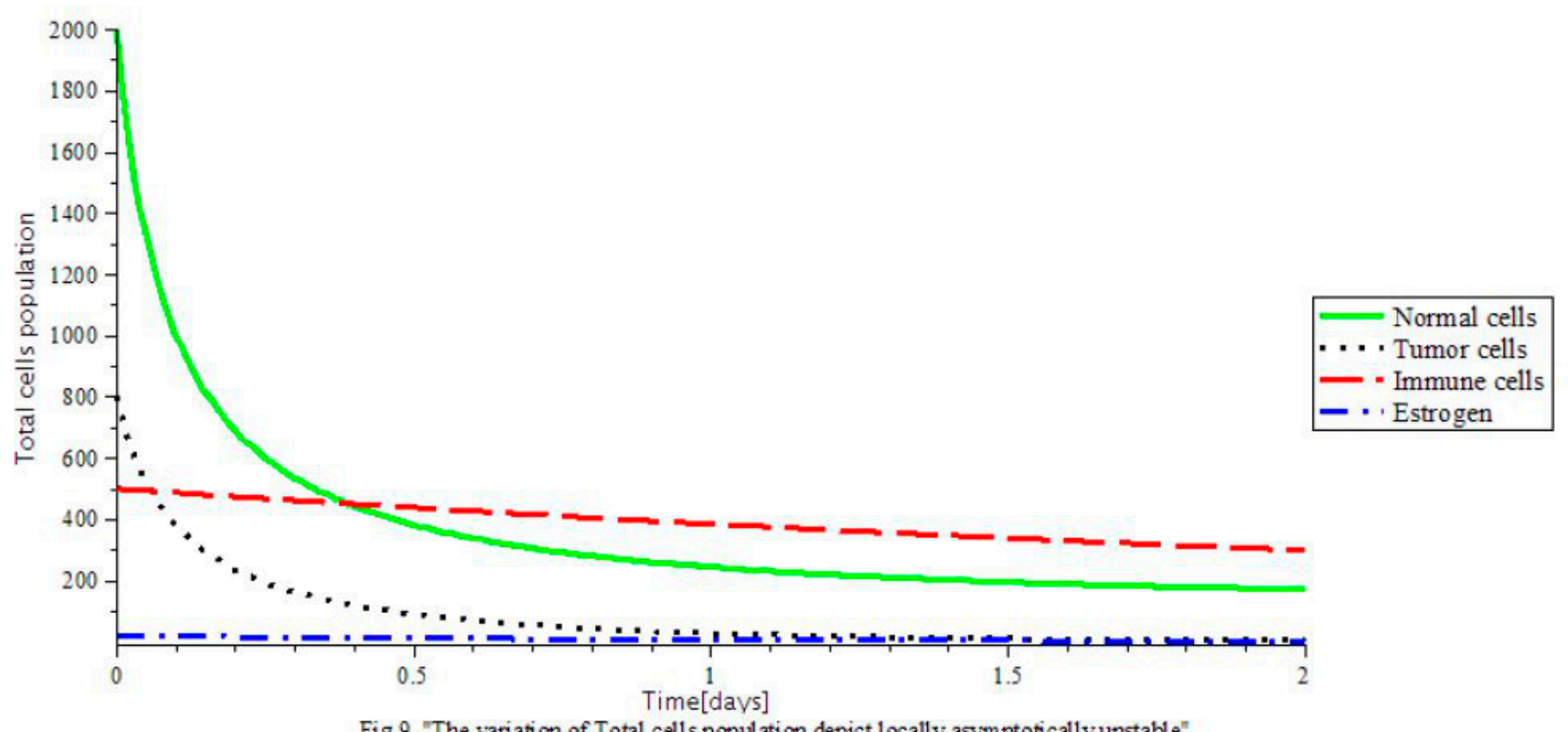

Figure 1.0 indicates that introduction of ketogenic diet result in reduction of activities of cancer cells and we also note that too much of ketogenic diet will result into ketoacidosis. Ketoacidosis is the combination of ketosis and acidosis; while ketosis is the accumulation of substances called ketone bodies and acidosis is increased acidity of the blood which can cause frequent urination (Polyuria),poor appetite and loss of consciousness. Therefore, our ketogenic diet's parameter rate is best at $d=0.6$ and it can compliment the activity of anti-cancer drug(Tamoxifen). Figure 2.0 shows the impact of anti-cancer drug in reducing the production of excess estrogen in the system, but when there is less production of estrogen there will not be rapid activation of growth factor that expresses breast normal cells. However, the rapid production of estrogen result in abnormal breast cells expression which will lead to breast cancer. While Figure 3.0 shows the effectiveness of anti-cancer drugs on tumor cells is very obvious because there is no supply of nutrient or glucose to cancer cells.

Furthermore, Figure 4.0 illustrates that, the red line $\beta=0$ is showing that during cancer formation the activities of both innate and adaptive reduces drastically due to the expression of other protein apart from those protein that are responsible for activation of immune response but as immune booster introduced to the system, it reactivate the activities of immune response towards the cancer cells.

The presence of abnormal estrogen level without anti-cancer drugs or ketogenic diet will lead the system into critical condition and became unstable as shown in Figure 7.0. However, the system became stable as we introduced the treatments such as chemotherapy and ketogenic diet as represented in Figure 6.0. Figure 5.1 indicates that there is DNA damage at $\lambda_{1}=0$ which occur naturally as a result of metabolic or hydrolytic processes. It is as a result of Tumor Suppressor Gene (TSG) is able to control the activity of DNA gene repair successfully. On the other hands, at $\lambda_{1}=0.2,0.4,0.6$ shown that TSG (such as BRCA 1,BRCA 2, P53) was compromised the 
pathway which leads to cells grow uncontrollably and later form a tumor or it also leads to accelerated aging.

However, the mathematical analysis of the model produced six equilibrium points. All the points have epidemiological implication in relation to explaining the breast cancer growth dynamics. $P_{0}$ represents the situation where there is tumor-free equilibrium that is when only tumor cell population has died off due to competition with other cells. $P_{d 1}$ represents Type 1dead equilibrium point where both normal cells and tumor cells are died off as a result of breast tissue removal through mastectomy surgery or death. This is because overtime the cancer cells which are depending on estrogen to develop into independent cells that grow regardless of estrogen receptors. $P_{d 2}$ could be described by Type 2-dead equilibrium point where normal cells only forced to extinction leaving the tumor cells surviving. $P_{d 3}$ represent Type 3-dead equilibrium point which means immune system is weak and it cannot fight the tumor cells which eventually overpower normal cells and forced it to extinction. $P_{d 4}$ show that Type 4-dead equilibrium point where ketogenic diet is not effective, immune booster is not active which lead to tumor cell over-compete normal cells as a result of infusion of excess estrogen to the body system.

We categorise this as a "dead" because biologically there is no recovery of damaged normal cells since they have been died off of the cell population. It could be as a result of anticancer drug that destroy red blood cells which affected normal cells. 


\subsection{Effects of Control on the system (52)}

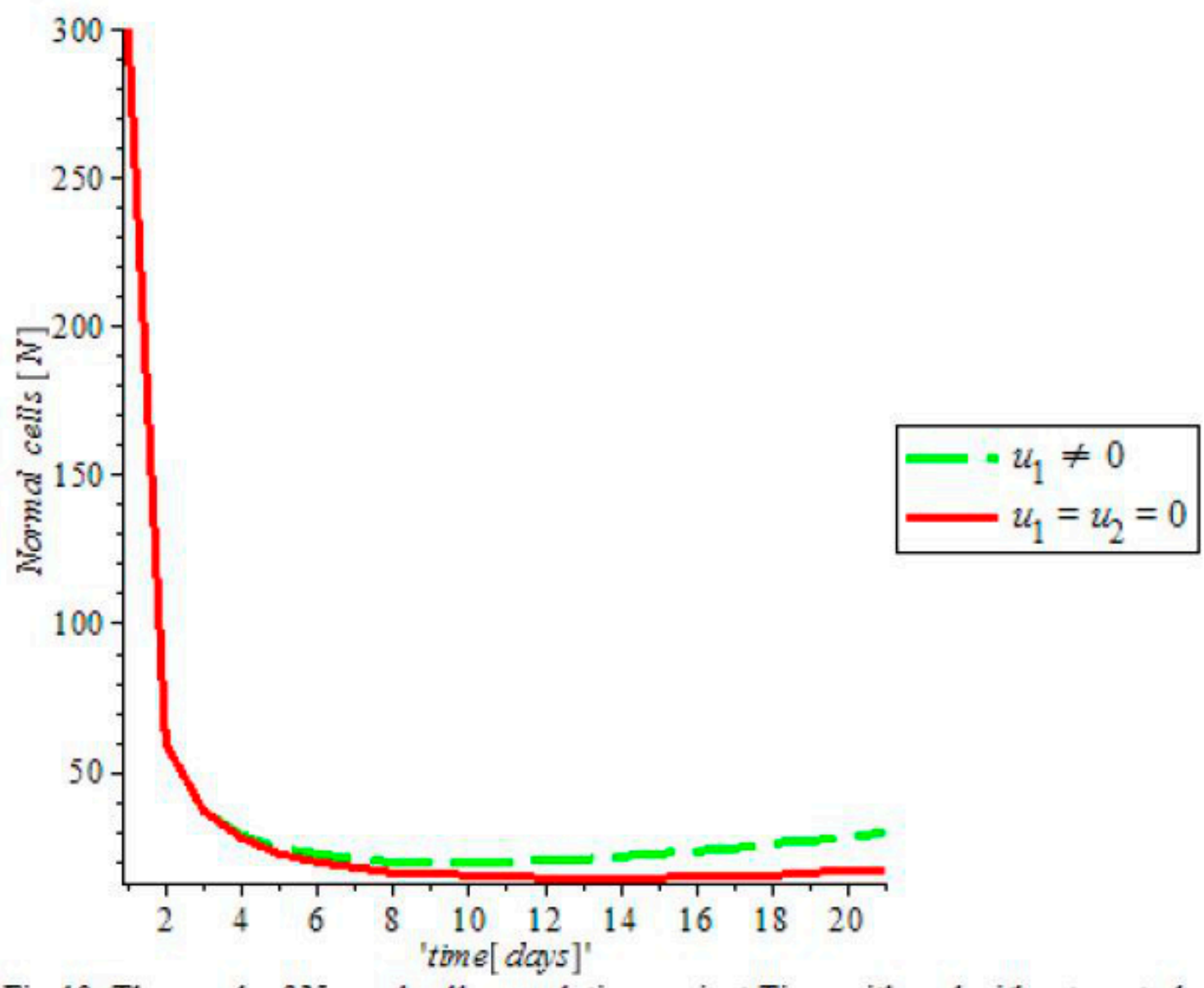

Fig 10: The graph of Normal cells population against Time with and without control

Effects of $u_{1}$ and $u_{2}$ on Tumor cells 


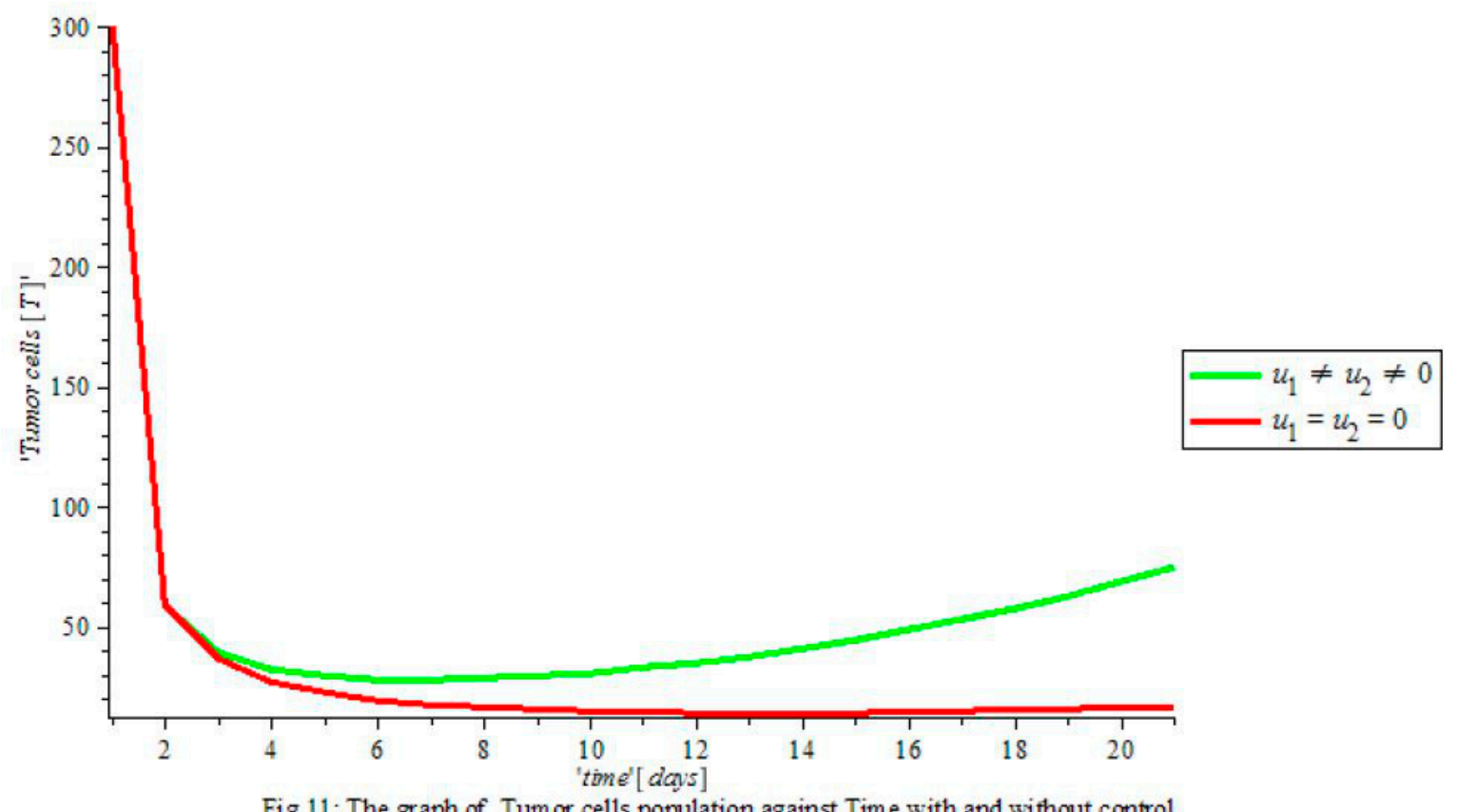

Fig 11: The graph of Tumor cells population against Time with and without control

Effects of $u_{1}$ on Estrogen

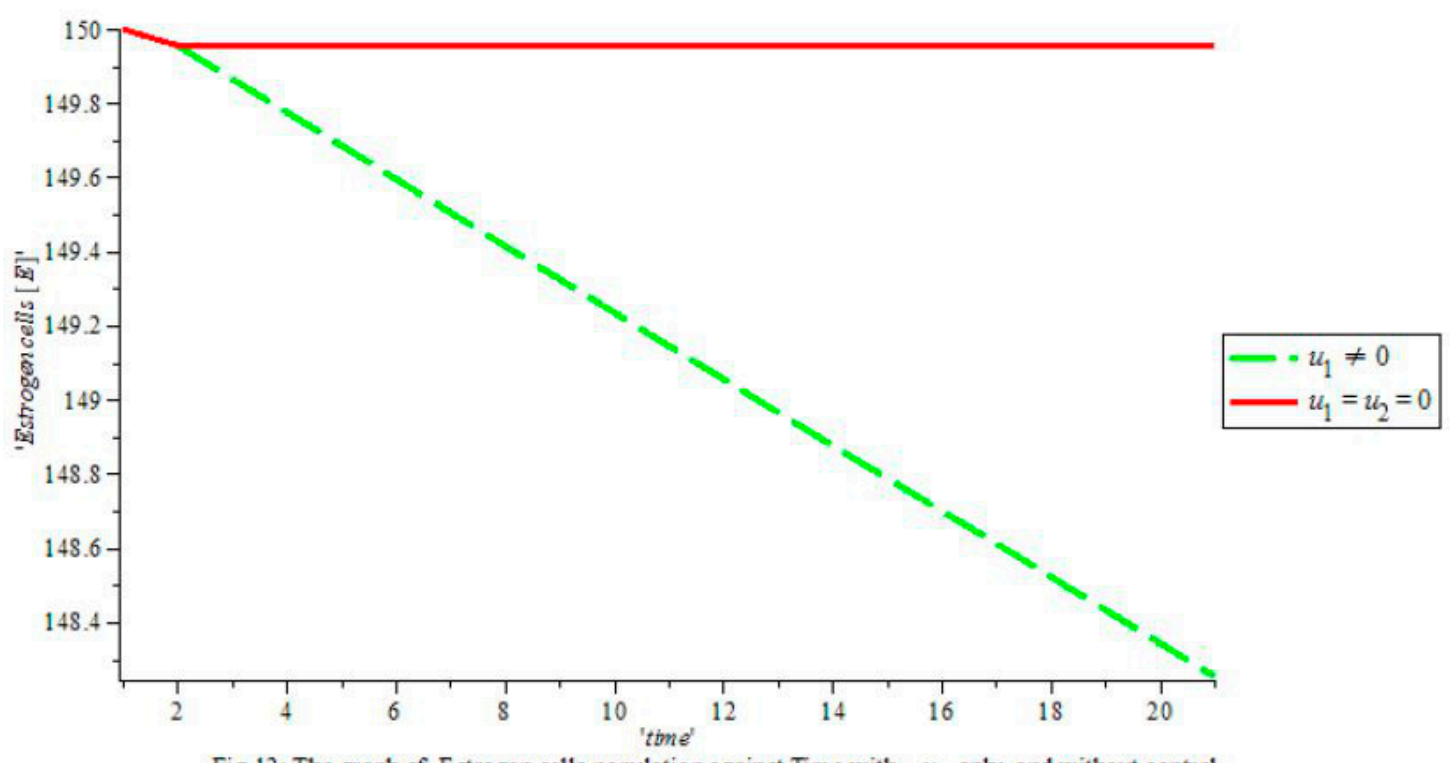

Fig 12: The graph of Estrogen cells population against Time with $u_{1}$ only and without control

Effects of $u_{2}$ on Estrogen 


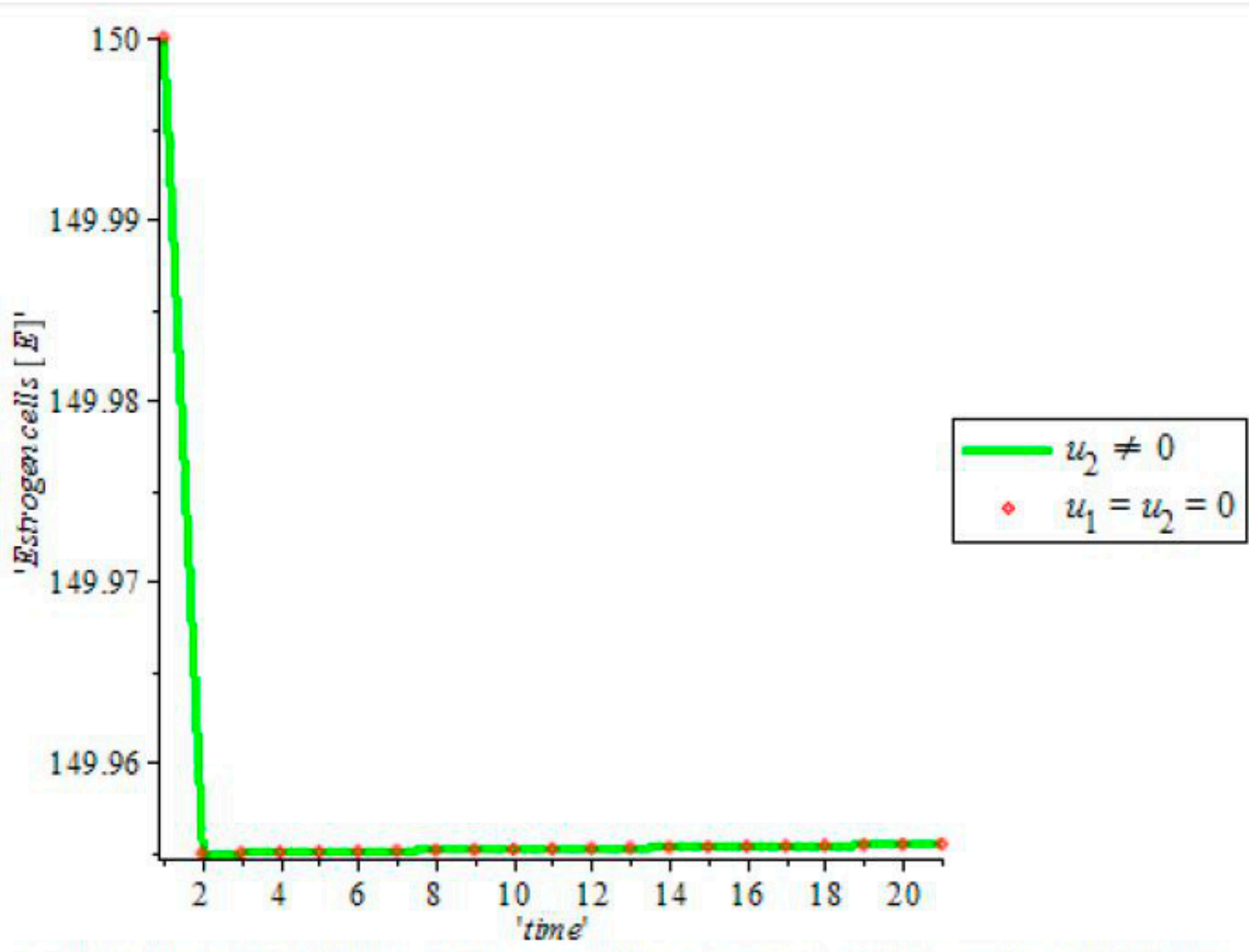

Fig 13: The graph of Estrogen cells population against Time with $u_{2}$ only and without control

In Figure 9.0, optimal control using anti-cancer drugs and ketogenic diet as we optimized the system (45) with the objective function $J$ for breast cancer model. It was observed that the combination of the two controls resulted in appreciable decrease in the number of tumor cells population (solid green line) compared to the increase recorded in the numbers of tumor cell population (solid red line) in the uncontrolled case. However, it was seeing in Figures $10 \& 11$, that the number of estrogen produced was drastically reduced using the two controls as against the constant increase observed in the number of estrogen produced in the uncontrolled cases (solid red line).

\section{Conclusion}

A four-dimensional compartmental deterministic model was designed and used to monitor dynamics of breast cancer. The existing model in [12] was extended to incorporate treatments, ketogenic diet and immune booster. The system (5) was rigorously analysed to gain insight into their dynamical behaviours. The study shows the following:

- conditions of stability of the Tumor-Free Equilibrium (TFE) was established and the system is only Local Asymptotically Stable (LAS) if a certain threshold quantity,known as reproductive number is less than unity $\left(R_{0}<1\right)$. It implies that the number of tumor cells in the 
body will be brought to zero if proper treatments and ketogenic diet which can make the threshold to a value less than unity are monitored.

- An individual has the chance of developing breast cancer depending on the level of immune system (s), efficacy of anti-cancer drug (k) and rate at which ketogenic diet (d) is being taken to fight tumor cells. We also found out that the presence of excess estrogen in system makes it unstable as depicted in Figure (1.0). This implies that any additional estrogen quantity introduced into the body through the birth control, Hormone Replacement Therapy (HRT) enhances rate of tumor formation. Thus, the development of breast cancer is certain.

- The transition from normal cells class to tumor cells class plays a crucial role in the breast cancer dynamics $\left(\lambda_{1}\right)$. More tumor formed if DNA is damage or altered as a result of excess estrogen; which reduces the number of normal cells being produced by red blood cells.

Furthermore, the results show that tumor cell formation depend on the level of excess estrogen introduced into the body system. It must be noted that, depending on an individual DNA's ability to resist changes in structure and amount of estrogen released during natural biological processes such as premenopause and menopause stages. Other risk factors may also be incorporated in the model for the future work which might generate different results.

However, the focus of this study has been identifying the advantages that come with the process of breast cancer relief policies that combine anti-cancer drugs and ketogenic diet procedures to knit the circumstances of unlimited and limited resources. The effort to moderate the effect of breast cancer on the body can be fruitful especially if our basic reproductive number $R_{0}$ is properly analysed. In addition, moderation is conceivable if the planning of intercessions is sufficiently quick and if the arrangement includes the utilization of more than one therapy procedure. No therapy ( ketogenic diet and anti-cancer drug) is possible unless minimal resources are accessible.

\section{References}

Abernathy, K., Abernathy, Z., Baxter, A. and Stevens, M., 2017. Global Dynamics of a Breast Cancer Competition Model. Differential Equations and Dynamical Systems, pp.1-15.

Allen, B.G., Bhatia, S.K., Anderson, C.M., Eichenberger-Gilmore, J.M., Sibenaller, Z.A., Mapuskar, K.A., Schoenfeld, J.D., Buatti, J.M., Spitz, D.R. and Fath, M.A., 2014. Ketogenic diets as an adjuvant cancer therapy: History and potential mechanism. Redox biology, 2, pp.963-970.

American Cancer Society. Breast Cancer,2013

De Pillis, L.G. and Radunskaya, A., 2001. A mathematical tumor model with immune resistance and drug therapy: an optimal control approach. Computational and Mathematical Methods in Medicine, 3 (2), pp.79-100.

De Pillis, L.G., Radunskaya, A.E. and Wiseman, C.L., 2005. A validated mathematical 
model of cell-mediated immune response to tumor growth. Cancer research, 65(17), pp.79507958.

Evans, C.W., 1991. The invasion and metastatic behaviour of malignant cells. The metastatic cell: behavior and biochemistry. London: Chapman and Hall, pp.137-214.

Gilbert, D.L., Pyzik, P.L. and Freeman, J.M., 2000. The ketogenic diet: seizure control correlates better with serum $\beta$-hydroxybutyrate than with urine ketones. Journal of child neurology, 15(12), pp.787-790.

Kareva, I. and Berezovskaya, F., 2015. Cancer immunoediting: a process driven by metabolic competition as a predator-prey-shared resource type model. Journal of theoretical biology, 380, pp.463-472.

Kumar, A. and Srivastava, P.K., 2017. Vaccination and treatment as control interventions in an infectious disease model with their cost optimization. Communications in Nonlinear Science and Numerical Simulation, 44, pp.334-343.

Lenhart, S. and Workman, J.T., 2007. Optimal control applied to biological models. Crc Press.

Marino, S., Hogue, I.B., Ray, C.J. and Kirschner, D.E., 2008. A methodology for performing global uncertainty and sensitivity analysis in systems biology. Journal of theoretical biology, 254(1), pp.178-196.

Mufudza Chipo, Walter Sorofa, and Edward T Chiyaka. Assessing the effects of estrogen on the dynamics of breast cancer. Computation and mathematical methods in medicine, 2012.

National Cancer Registry of South Africa. 2010

Pontryagin, L.S., 1987. Mathematical theory of optimal processes. CRC Press.

Patel, M.I. and Nagl, S., 2010. The role of model integration in complex systems modelling: An example from cancer biology. Springer.

Pinho, S.T.R.D., Freedman, H.I. and Nani, F., 2002. A chemotherapy model for the treatment of cancer with metastasis. Mathematical and Computer Modelling, 36(7-8), pp.773803.

Valayannopoulos, V., Bajolle, F., Arnoux, J.B., Dubois, S., Sannier, N., Baussan, C., Petit, F., Labrune, P., Rabier, D., Ottolenghi, C. and Vassault, A., 2011. Successful treatment of severe cardiomyopathy in glycogen storage disease type III With D, L-3-hydroxybutyrate, ketogenic and 
high-protein diet. Pediatric research, 70(6), pp.638-641.

Westman, E.C., Yancy, W.S., Mavropoulos, J.C., Marquart, M. and McDuffie, J.R., 2008. The effect of a low-carbohydrate, ketogenic diet versus a low-glycemic index diet on glycemic control in type 2 diabetes mellitus. Nutrition and metabolism, 5(1), p.36.

Wu, C.H., Motohashi, T., Abdel-Rahman, H.A., Flickinger, G.L. and Mikhail, G., 1976. Free and protein-bound plasma estradiol $-17 \beta$ during the menstrual cycle. The Journal of Clinical Endocrinology and Metabolism, 43(2), pp.436-445.

World Health Organization. Global action plan for the prevention and control on NCDs. 2014

Perko, L., 2013. Differential equations and dynamical systems (Vol. 7). Springer Science and Business Media.

Malinzi, J., Eladdadi, A. and Sibanda, P., 2017. Modelling the spatiotemporal dynamics of chemovirotherapy cancer treatment. Journal of Biological Dynamics, 11(1), pp.244-274.

d'Onofrio, A., Ledzewicz, U., Maurer, H. and Schättler, H., 2009. On optimal delivery of combination therapy for tumors. Mathematical biosciences, 222(1), pp.13-26.

Bernoulli, D., 1760. Essai d'une nouvelle analyse de la mortalité causée par la petite vérole et des avantages de l'inoculation pour la prévenir. Histoire de l'Acad. Roy. Sci.(Paris) avec Mém. des Math. et Phys. and Mém, 1, pp.1-45.

Kermack, W.O. and McKendrick, A.G., 1927, August. A contribution to the mathematical theory of epidemics. In Proceedings of the Royal Society of London A: mathematical, physical and engineering sciences (Vol. 115, No. 772, pp. 700-721). The Royal Society.

Kermack, W.O. and McKendrick, A.G., 1939. Contributions to the mathematical theory of epidemics: V. Analysis of experimental epidemics of mouse-typhoid; a bacterial disease conferring incomplete immunity. Epidemiology \& Infection, 39(3), pp.271-288.

Gatenby,R.A.,1995. The potential role of transformation-induced metabolic changes in tumor-host interaction. Cancer Research, Vol 1766, No.2, pp.4151-4156.

Neves, A.A and Brindle, K.M., 2006. Assessing responses to cancer therapy using molecular imaging. Biochimica et Biophysica Acta, Vol. 1766, no. 2, pp.242-261 
Schattler, H. and Ledzewicz, U., 2015. Optimal control for mathematical models of cancer therapies. Springer New York.

Swierniak, A., Krzeslak, M., Student, S. and Rzeszowska-Wolny, J., 2016. Development of a population of cancer cells: observation and modeling by a mixed spatial evolutionary games approach. Journal of theoretical biology, 405, pp.94-103.

Kimmel, M. and Swierniak, A., 2006. Control theory approach to cancer chemotherapy: Benefiting from phase dependence and overcoming drug resistance. In Tutorials in mathematical biosciences III (pp. 185-221). Springer, Berlin, Heidelberg. 